\title{
Development of a thiophene derivative modified LDH coating for Mg alloy corrosion protection.
} LI, L.-X., XIE, Z.-H., FERNANDEZ, C., WU, L., CHENG, D., JIANG, X.-H., ZHONG., C.-J. 


\section{Journal Pre-proof}

Development of a thiophene derivative modified LDH coating for Mg alloy corrosion protection

Lin-Xin Li, Zhi-Hui Xie, Carlos Fernandez, Liang Wu, Daojian Cheng, Xiao-Hui Jiang, Chuan-Jian Zhong

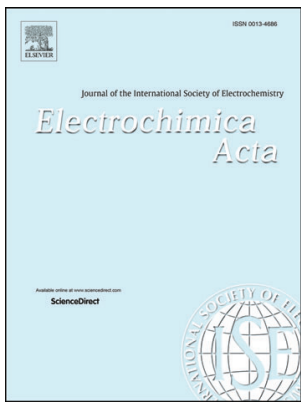

PII:

S0013-4686(19)32057-2

DOI:

https://doi.org/10.1016/j.electacta.2019.135186

Reference: $\quad$ EA 135186

To appear in: Electrochimica Acta

Received Date: 16 September 2019

Revised Date: 28 October 2019

Accepted Date: 31 October 2019

Please cite this article as: L.-X. Li, Z.-H. Xie, C. Fernandez, L. Wu, D. Cheng, X.-H. Jiang, C.-J. Zhong, Development of a thiophene derivative modified LDH coating for Mg alloy corrosion protection, Electrochimica Acta (2019), doi: https://doi.org/10.1016/j.electacta.2019.135186.

This is a PDF file of an article that has undergone enhancements after acceptance, such as the addition of a cover page and metadata, and formatting for readability, but it is not yet the definitive version of record. This version will undergo additional copyediting, typesetting and review before it is published in its final form, but we are providing this version to give early visibility of the article. Please note that, during the production process, errors may be discovered which could affect the content, and all legal disclaimers that apply to the journal pertain.

(c) 2019 Published by Elsevier Ltd. 

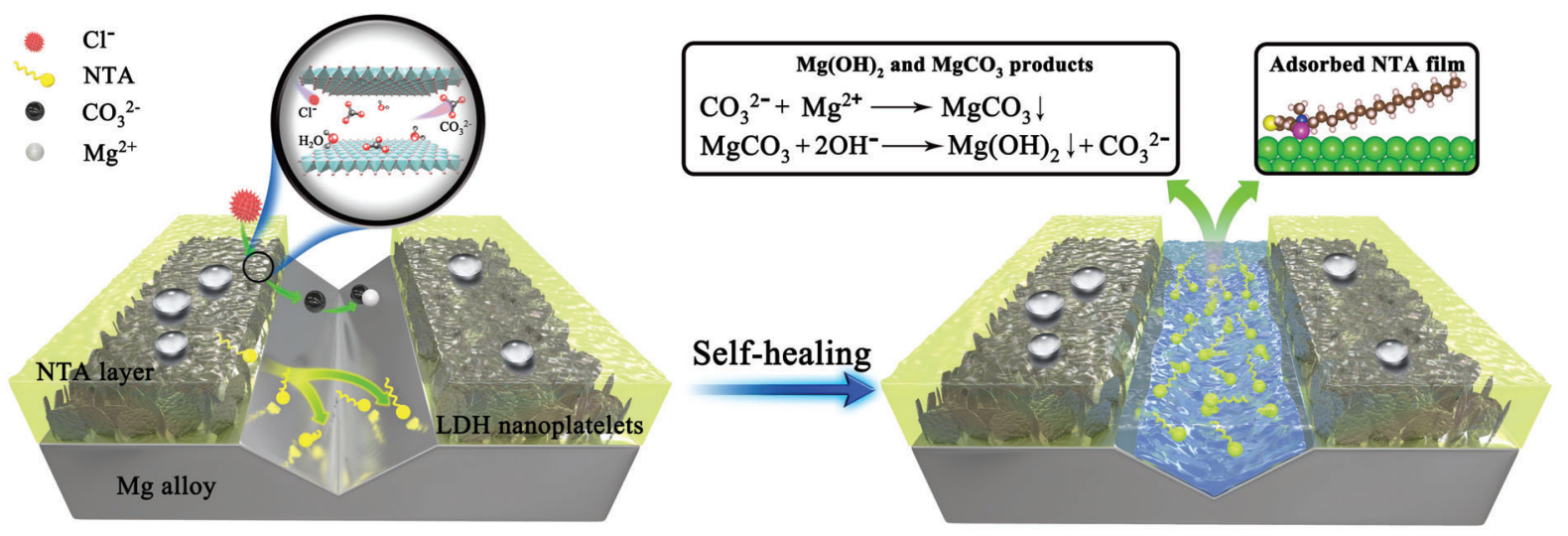


\title{
Development of a Thiophene Derivative Modified LDH Coating for Mg Alloy Corrosion Protection
}

Lin-Xin Li ${ }^{\mathrm{a}}$, Zhi-Hui Xie ${ }^{\mathrm{a}, \mathrm{e},{ }^{*}}$, Carlos Fernandez ${ }^{\mathrm{b}}$, Liang $\mathrm{Wu}^{\mathrm{c}}$, Daojian Cheng ${ }^{\mathrm{d}}$, Xiao-Hui Jiang ${ }^{\mathrm{a}}$, Chuan-Jian Zhong ${ }^{\mathrm{e}}$

${ }^{a}$ Chemical Synthesis and Pollution Control Key Laboratory of Sichuan Province/Institute of Synthesis and Application of Functional Materials, College of Chemistry and Chemical Engineering, China West Normal University, Nanchong, 637002, P.R. China.

${ }^{\mathrm{b}}$ School of Pharmacy and Life Sciences, Robert Gordon University, Garthdee Road, Aberdeen, AB107GJ United Kingdom

${ }^{\mathrm{c}}$ College of Materials Science and Engineering/National Engineering Research Center for Magnesium Alloys, Chongqing University, Chongqing 400044, P.R. China

${ }^{\mathrm{d}}$ Key Laboratory of Organic-Inorganic Composites, Beijing University of Chemical Technology, Beijing 100029, P.R. China

${ }^{e}$ Department of Chemistry, State University of New York at Binghamton, Binghamton, New York 13902, United States

Corresponding Author: E-mail: zhxie@cwnu.edu.cn; zxie@binghamton.edu (Z.-H. Xie)

\begin{abstract}
A new thiophene derivative corrosion inhibitor (CI) was used to functionalize the layered double hydroxide (LDH) primer on $\mathrm{Mg}$ alloy to enhance corrosion protection. Compared with bare LDH coating, the composite coatings consisting of a MgAl-LDH primer and an outer CI layer showed highly compact surface structures without large pores and gaps, thereby indicating the enhanced capability to resist the penetration of corrosive species. Eelectrochemical tests revealed that the MgAl-LDH film modified with the newly synthesized CI exhibited higher charge transfer resistance and lower corrosion current density than films modified with commercial CIs. The highest enhancement in corrosion resistance was linked to the film's ability to repair physical damage by forming precipitates through chemical complexation and adsorption of the CIs. These findings open up new opportunities for the design and synthesis of a thiophene-contained inhibitor to modify LDH films and to obtain a composite coating with superior corrosion protection.
\end{abstract}

KEYWORDS: Mg alloy; corrosion; coating; layered double hydroxide 


\section{INTRODUCTION}

Magnesium (Mg) alloys have attracted great research interest and has been applied to many fields ranging from communication systems, fuel-efficient vehicles, medical implants, battery components, and aerospace industry, owing to its intrinsic merits, such as extremely low density, high specific strength, and good cast ability [1-4]. A shortcoming of Mg alloys is their susceptibility to corrosion, especially in humid environments containing $\mathrm{Cl}^{-}$, and $\mathrm{SO}_{4}{ }^{2-}$, due to their high chemical activity, small Pilling-Bedworth ratio (PBR) of $\mathrm{MgO}$ to substrate $(\mathrm{PBR}=0.81)$, and low standard electrode potentials, i.e., around $-1.5 \mathrm{~V}$ vs. saturated calomel electrode. This corrosion susceptibility would eventually lead to a loose and incompact oxide film without protective function, continuous hydrogen evolution reaction, and oxidation of $\mathrm{Mg}$ [5-7]. The most effective way to isolate the substrate from the corrosive medium by forming a complete physical barrier, namely coating, through different methods [8]. Many coatings have been developed through various approaches, e.g., chemical conversion films $[9,10]$, ceramic membranes developed by microarc oxidation [11, 12], metallic coatings developed by electroless plating and electroplating [13-16], and organic coatings developed by sol-gel method [17, 18]. Except for chromium conversion film that has been strictly restricted or even prohibited in many countries because of its adverse effects on humans and the environment, an issue of conventional coatings is the deficiency in self-healing functionality when occurrence of defects and corrosion.

Layered double hydroxides (LDHs), which are widely applied in industry and have attracted significant research interest, were fabricated as coatings for corrosion protection in the last decade to mimic the self-repairing behavior of traditional Cr conversion films by utilization of their good ion exchangeability $[19,20]$. As affinities of anions for LDH intercalation differ, aggressive ions can be trapped into the interlayers, whereas intercalated anions are released and serve as inhibitors that increase the corrosion protection of the LDH coating [20, 21]. The released anions may react with the $\mathrm{Mg}^{2+}$ that results from the oxidation of $\mathrm{Mg}$ alloys, thereby filling the corroded sites by producing compact and stable depositions, modifying the defects, decreasing the aggressiveness of the environment, and showing a self-healing ability $[22,23]$. However, LDH nanosheets are usually grown randomly and perpendicularly to the substrate surface with a nest-like morphology, thereby creating a porous film with many open pores and 
gaps regularly extending to the matrix/LDH film interface, which supplies diffusion pathways for corrosive species and undermines the corrosion protection capability of LDH coatings [19]. There are two strategies to reduce the negative effect of the inherent microporous structure of LDH coatings. One strategy involves the intercalation of different inorganic and organic inhibitors, such as vanadate, into the interlayer galleries of LDHs through the anion-exchange process $[24,25]$. The intercalation of corrosion inhibitors (CIs) results in the reduction of average sized crystallites and the formation of more compact LDH film through basal spacing expansion $[24,26]$. Another strategy is immersion in a solution containing different inhibitors to form a new protective film on the LDH surface, which can both seal the micropores and act as the first physical barrier; the new film can show other functions such as superhydrophobicity [27-30]. For instance, Yao et al. prepared a MgAl-LDH film modified with a mixture of methyltrimethoxysilane and cerium nitrate on AZ31 Mg alloy. The newly formed film consisted of polymethyltrimethoxysilane and $\mathrm{CeO}_{2}$, which showed satisfactory self-cleaning behavior. Such behavior is due to the formation of a superhydrophobic surface originating from aliphatic chains of silanes and self-healing ability originating from the precipitations of $\mathrm{MgCO}_{3}$ and $\mathrm{Mg}(\mathrm{OH})_{2}$, thereby resulting in the long-term service of the Mg alloy [28]. Inhibitor properties play the most important role regardless of the strategy used (intercalation or immersion). However, to the best of our knowledge, all the inhibitors used for modification of LDH film on Mg alloys in the literature, including inhibitors adopted in some of our recent publications, such as stearic acid, myristic acid, 8-quinolinol, and others [22, 30]. are purchased from commercial companies instead of synthesized by the authors themselves.

In this study, a MgAl-LDH coating modified with a new synthesized thiophene derivative inhibitor, namely N-alkyl-N, N-dimethyl-N-(3-thienylmethylene) ammonium bromides (NTA), was fabricated on an AZ31 Mg alloy matrix through a hydrothermal method followed by a simple immersion step. This coating shows a significant enhancement in corrosion resistance. The as-obtained coatings consisted of an underlying MgAl-LDH layer, as confirmed by high angle annular dark-field scanning transmission electron microscopy (HAADF-STEM) images, energy dispersive X-ray spectroscopy (EDS), and X-ray diffractometer (XRD). An outer layer was created by the adsorption of CIs, which was confirmed by X-ray photoelectron spectroscopy and electron probe micro-analyzer. In addition to the physical barrier of the 
coating, the significant boost in corrosion protection of the NTA-modified MgAl-LDH system was due to the self-repair of physical damage (self-healing process) through the following reactions: (a) precipitations produced from chemical reactions of $\mathrm{Mg}^{2+}$, i.e., the oxidation of $\mathrm{Mg}$ and the release of $\mathrm{CO}_{3}{ }^{2-}$ from $\mathrm{LDH}$ by ion exchange process; and (b) physical and chemical adsorption of NTA molecules on the exposed Mg surface. The latter assumption was confirmed by theoretical calculation of the adsorption energy $\left(E_{\mathrm{a}}\right)$ of NTA on the Mg (0001) surface using density functional theory (DFT). For comparison, many characterizations and electrochemical tests of MgAl-LDH coatings modified by several commercial CIs were also given and analyzed.

\section{EXPERIMENTAL SECTION}

\subsection{Materials and Preparation of Different MgAl-LDH Coatings}

The chemicals used to prepare the MgAl-LDH coating and the CIs@MgAl-LDH coatings were acquired from Aladdin Industrial Inc. or Sinopharm Chemical Reagent Co., Ltd. (Shanghai, China), and NTA was synthesized in our laboratory. The Mg alloy was first ground with a 1200 grit sandpaper to form a mirror-like surface and then washed with an alkali solution containing $50 \mathrm{~g} / \mathrm{L}$ of $\mathrm{NaOH}(\geq 98.0 \%)$ and 10 $\mathrm{g} / \mathrm{L}$ of $\mathrm{Na}_{3} \mathrm{PO}_{4}(\geq 98.0 \%)$ for $10 \mathrm{~min}$. The solution for the deposition of the MgAl-LDH film was prepared by mixing $1.539 \mathrm{~g}$ of $\mathrm{Mg}\left(\mathrm{NO}_{3}\right)_{2} \cdot 6 \mathrm{H}_{2} \mathrm{O}(\geq 99.0 \%), 0.750 \mathrm{~g}$ of $\mathrm{Al}\left(\mathrm{NO}_{3}\right)_{3} \cdot 9 \mathrm{H}_{2} \mathrm{O}(\geq 99.0 \%)$, and $0.106 \mathrm{~g}$ of $\mathrm{Na}_{2} \mathrm{CO}_{3}(\geq 99.8 \%)$ in $100 \mathrm{~mL}$ of ultrapure water at $25^{\circ} \mathrm{C}$ followed by a pH adjustment to 12 by using the $\mathrm{NaOH}$ solution. Afterward, the treated $\mathrm{Mg}$ alloy was vertically placed in a $50 \mathrm{~mL}$ Teflon-lined autoclave, and then, the abovementioned mixture was added. The autoclave was capped and transferred into an oven for reactions at $125{ }^{\circ} \mathrm{C}$ for $24 \mathrm{~h}$. Finally, the $\mathrm{Mg}$ alloy was taken out, rinsed with water, and dried in a vacuum at $65{ }^{\circ} \mathrm{C}$ for $10 \mathrm{~h}$. The obtained sample was denoted by a MgAl-LDH coating. To prepare the MgAl-LDH coating treated by different CIs, $1.0 \mathrm{~g}$ of 2-mercaptobenzothiazole (MBT, 98\%), $0.15 \mathrm{~g}$ of sodium alginate (SA, CP), and $1.0 \mathrm{~g}$ of sodium dodecyl benzene sulfonate (SDBS, 95\%) were dissolved in $25 \mathrm{~mL}$ ultrapure water, followed by adjustment of the $\mathrm{pH}$ to 12 by using the $\mathrm{NaOH}$ solution. To improve the solubility, NTA $(0.15 \mathrm{~g})$ was dissolved in ethanol $(75 \mathrm{vol} . \%)$ at the same volume. Then, MgAl-LDH samples were soaked in these CI solutions at $25{ }^{\circ} \mathrm{C}$ for $48 \mathrm{~h}$. The obtained samples were rinsed with 
distilled water and marked as MBT@MgAl-LDH, SA@MgAl-LDH, SDBS@MgAl-LDH, and NTA@MgAl-LDH coatings. For convenience of description, CIs@MgAl-LDH, which stands for all the MgAl-LDH samples that have been treated by different CIs, is also used in the manuscript. The schematic graphs in Scheme 1a-c show the deposition of the MgAl-LDH film and the preparation of the CIs@MgAl-LDH coatings.

\subsection{Synthesis and characterization of NTA}

$\mathrm{N}$, N-dimethylalkylamine (0.012 mol, $98 \%)$ was first dissolved in acetonitrile ( $8 \mathrm{~mL}, \geq 99.5 \%)$ in an oil bath at $55{ }^{\circ} \mathrm{C}$. Then, an acetonitrile mixture $(10 \mathrm{~mL})$ containing 3-bromomethylthiophene $(0.01 \mathrm{~mol}, 97 \%)$ was added drop wise to the abovementioned solution, and the refluxed reaction was continued $24 \mathrm{~h}$. Afterward, the solvent was distilled under reduced pressure, and the raw products were purified by column chromatography on silica gel [column length: about $12 \mathrm{~cm}$, methanol (99.5\%): methylene chloride (99.5\%) $=1: 10$ ]. Finally, the obtained sample was recrystallized [ethyl acetate $(99.5 \%)$ : dichloromethane (99.5\%) =10: 1)]. The final sample was NTA. The final product was characterized by ${ }^{1} \mathrm{H}$ NMR, ${ }^{13} \mathrm{C}$ NMR, MS, and Fourier infrared spectrometer (FT-IR) to determine and ascertain the structure. The corresponding steps for NTA synthesis and the chemical reaction are depicted in Scheme 1d.
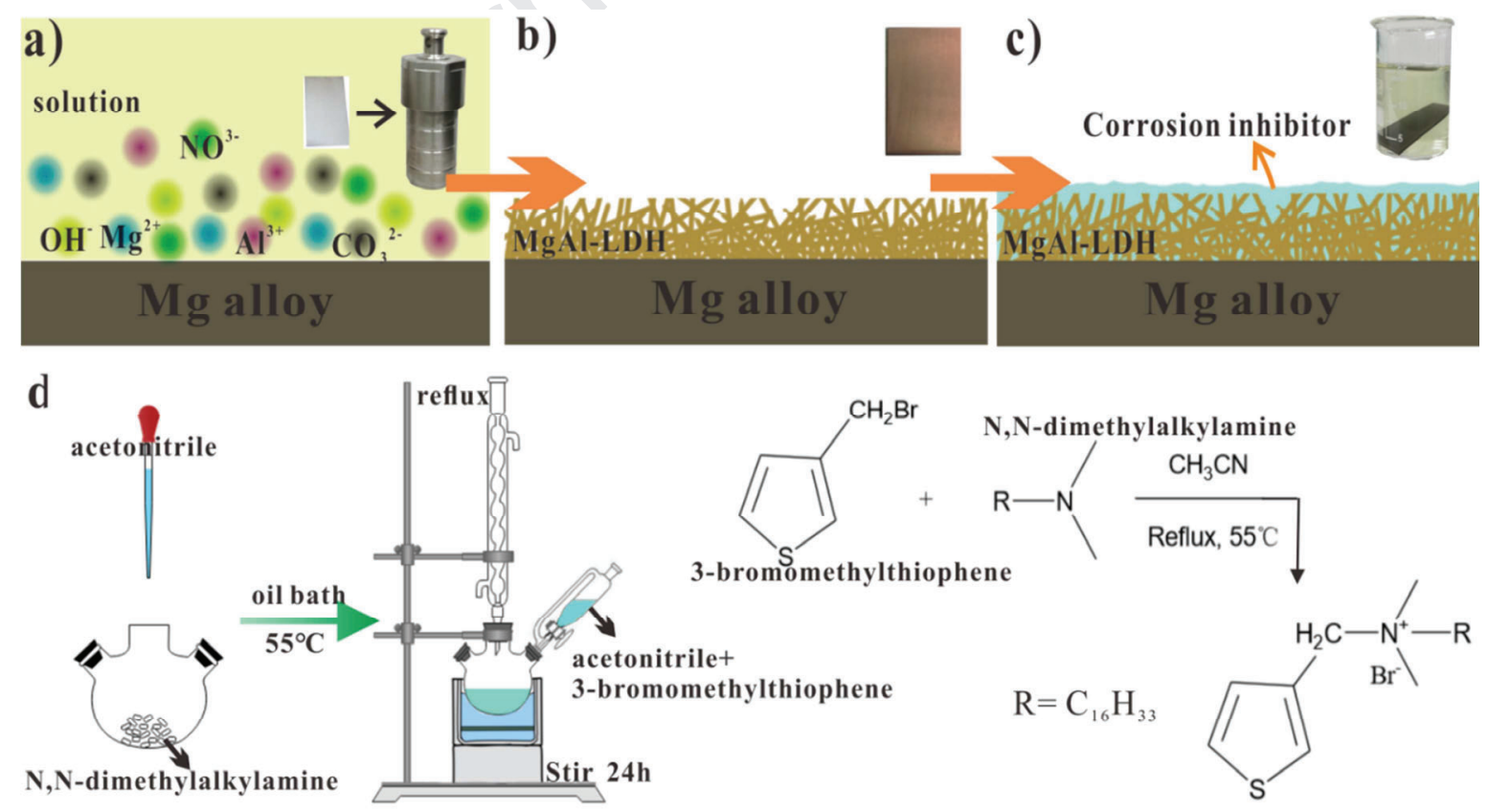

Scheme 1. The schematic diagrams of the (a, b, and c) in-situ growth of MgAl-LDH film and the preparation of CIs@MgAl-LDH coatings on Mg alloy and (d) synthesis of NTA inhibitor. 


\subsection{Morphology and Structure Characterization}

XRD (D8 Advance, $\mathrm{Cu}$ target, incident wavelength $\lambda=0.154 \mathrm{~nm}$ ) was used to characterize the crystal structure of the coatings. X-ray photoelectron spectroscopy (XPS, Thermo ESCALAB 250XI, C 1s = $284.8 \mathrm{eV}$ ) was used to test the chemical composition of the coating surface. The functional groups of the coating were acquired using FT-IR (Nicolet-6700, 400 4000 $\mathrm{cm}^{-1}$ ). The surface morphology, composition, and structure of the coating were observed by scanning electron microscopy (SEM, SU8020, $3 \mathrm{kV}$ ) and transmission electron microscope (TEM, Tecnai G2 F20, $2000 \mathrm{kV}$ ). The distribution and contents of various elements on the surface of the samples were characterized by electron probe micro-analyzer (EPMA, JXA-8230, $15 \mathrm{kV}, 10 \mathrm{nA})$ and EDS coupled with TEM. Proton nuclear magnetic resonance $\left({ }^{1} \mathrm{H}\right.$ NMR, Bruker-400 nuclear magnetic resonance spectrometer, $400 \mathrm{MHz}, \mathrm{CDCL}_{3}$, TMS), Carbon-13 nuclear magnetic resonance spectrometry $\left({ }^{13} \mathrm{C}\right.$ NMR, Bruker-400 nuclear magnetic resonance spectrometer, 100 $\mathrm{MHz}, \mathrm{CDCL}_{3}$, TMS), mass spectrum (Bruker micrOTOF-Q ), and FT-IR were used to ascertain the chemical structure of NTA.

\subsection{Scratch Test}

Half of the MgAl-LDH sample was immersed in an NTA solution, thereby exposing the remaining area of the coating to air for $48 \mathrm{~h}$. After the immersion step, a knife was used to cut the surface across both NTA-free and NTA-treated areas to form a scratch with a depth that was sufficient to reach the substrate. Then, the entire sample was immersed in $3.5 \mathrm{wt} . \% \mathrm{NaCl}$ solution. The evolution of the scratches on the surface was observed and analyzed by SEM images and EDS spectra.

\subsection{Electrochemical Test}

The electrochemical experiment was carried out by a Gamry electrochemical workstation (Interface 1010E) in $\mathrm{NaCl}$ solution (3.5 wt.\%) at $25^{\circ} \mathrm{C}$. A classical three-electrode system was used. The reference electrode was a saturated silver chloride electrode $(\mathrm{Ag} / \mathrm{AgCl})$. The auxiliary electrode was a platinum electrode. The prepared sample with an exposed area of $1 \mathrm{~cm}^{2}$ was used as the working electrode. The EIS test frequencies ranged from $0.01 \mathrm{~Hz}$ to $100 \mathrm{kHz}$ with an excitation signal amplitude of $10 \mathrm{mV}$, and the EIS data were processed by ZsimpWin software. The potential range during potentiodynamic polarization tests was from -0.3 to $+0.3 \mathrm{~V}$ vs. open circuit potential, and the scan rate was $5 \mathrm{mV} / \mathrm{s}$. The electrolytic cell 
was placed in a Faraday cage to shield against external electromagnetic interference. Before the formal electrochemical tests, the samples were immersed in the $\mathrm{NaCl}$ solution for approximately 20 min to obtain a potential plateau.

\subsection{DFT Calculations [31, 32]}

All calculations were carried out by using the projector augmented wave method in the framework of spin-polarized the DFT, as implemented in the Vienna ab-initio Simulation Package. The generalized gradient approximation and Perdew-Burke-Ernzerhof exchange function were used. The plane-wave energy cutoff was set to $500 \mathrm{eV}$, and the Monkhorst-Pack method was used for the Brillouin zone sampling. The convergence criteria of energy and force calculations were set to $10^{-5} \mathrm{eV} /$ atom and $0.01 \mathrm{eV}$ $\AA^{-1}$, respectively. The $\mathrm{Mg}(0001)$ slab was built from its $6 \sqrt{3} \times 4$ supercell of the $(0001)$ surface with a vacuum of $15 \AA$.

\section{RESULTS and DISCUSSION}

The typical SEM and TEM micrographs of the MgAl-LDH precipitation are presented in Figure 1. The SEM images (Figure 1a-b) show that many flake-like nanosheets with thickness values of approximately $20 \mathrm{~nm}$ and lengths ranging from 1000 to $100 \mathrm{~nm}$ are formed on the Mg alloy surface. Meanwhile, many gaps between nanoplatelets were also found, which helped increase the area of the surface and the adsorption of more CIs. The flake-like structure of the MgAl-LDH film is also verified by the TEM image, as shown in Figure 1c. The image shows several overlapped nanoplatelets with widths of approximately $250 \mathrm{~nm}$. The HAADF-STEM images and corresponding EDS elemental maps as given in Figure 1d-f show the full and uniform distributions of $\mathrm{Mg}$ and $\mathrm{Al}$ elements with a $\mathrm{Mg} / \mathrm{Al}$ atomic ratios of approximately 3 within the MgAl-LDH nanosheets, in accordance with the molecular formula of MgAl-LDH deposition $\left(\mathrm{Mg}_{6} \mathrm{Al}_{2}\left(\mathrm{CO}_{3}\right)(\mathrm{OH})_{16} \cdot 4 \mathrm{H}_{2} \mathrm{O}\right.$, JCPDS NO: 41-1428), thereby indicating the successful growth of MgAl-LDH film on the Mg alloy surface. There reference meaning for the ratio of $\mathrm{C}$ and $\mathrm{O}$ was not much because of the ubiquitous contamination of $\mathrm{C}$ and unreliability of the EDS results for light elements $(\mathrm{Z}<11)$. The contents of $\mathrm{C}$ and $\mathrm{O}$ merely qualitatively indicated the existence of the corresponding elements in the nanosheets. The $\mathrm{Cu}$ comes from the copper grids found in the supporting sample. 

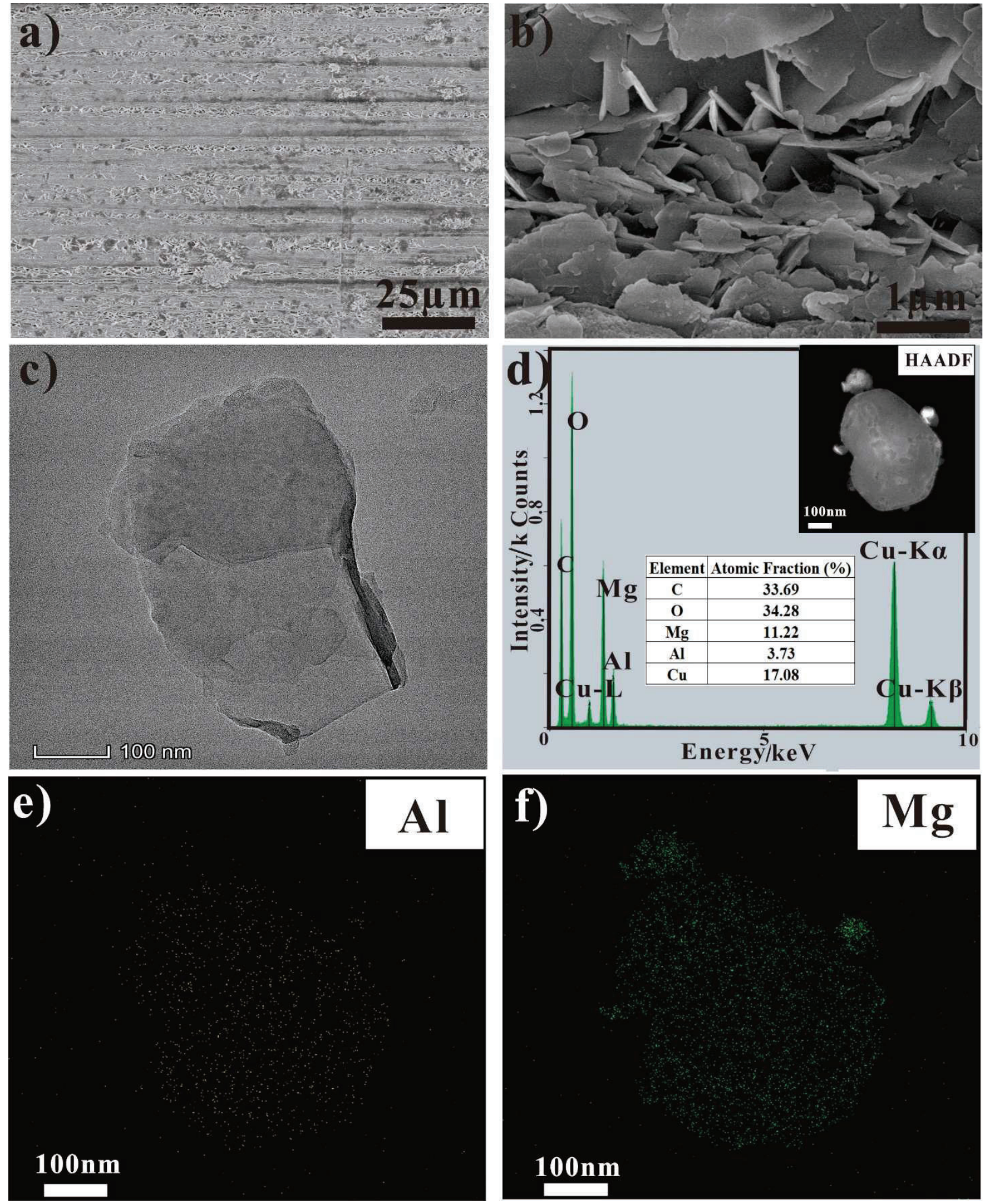

Figure 1. (a, b) SEM and (c) TEM of MgAl-LDH depositions, and (d, e, f) the corresponding HAADF-STEM-EDS spectra and maps showing the full and uniform distribution of $\mathrm{Al}$ and $\mathrm{Mg}$.

According to results and analyses of the ${ }^{1} \mathrm{H}$ NMR, ${ }^{13} \mathrm{C}$ NMR, MS, and FT-IR spectra (given in the Support Information, Figs. S1-S4 and Tables S1-S3), the NTA was synthesized successfully. The surface 
morphologies of the MgAl-LDH coating after modification by the as-synthesized NTA are shown in

Figure 2. The MgAl-LDH coatings modified with several common commercial CIs, including MBT, SA, and SDBS, using the same steps and conditions are also given for comparision. The SEM images in Figure 2 show that the perpendicular growth nanosheets on the substrate (Figure 1b) are covered by many new structures after CI modification. For example, many of these structures are aligned in a parallel manner with irregular nanosheets. Many silk-like species are observed on the surface of the MBT@MgAl-LDH coating (Figure 2a-c), whereas NTA@MgAl-LDH coating exhibit a more compact surface with some coral-like structures (Figure $\mathbf{2} \mathrm{j}-1$ ), thereby showing the formation of CI films on the MgAl-LDH coating surfaces. After treatment with CIs, the big pores and gaps in the MgAl-LDH coating that may serve as corrosion paths during exposure to a corrosive environment are totally covered, and a compact and dense surface is formed by the adsorption of CIs, thereby leading to the delayed penetration of corrosive medium. Compared with bare MgAl-LDH coating, the formations of compact CI films provided better corrosion protection to the $\mathrm{Mg}$ alloy. 

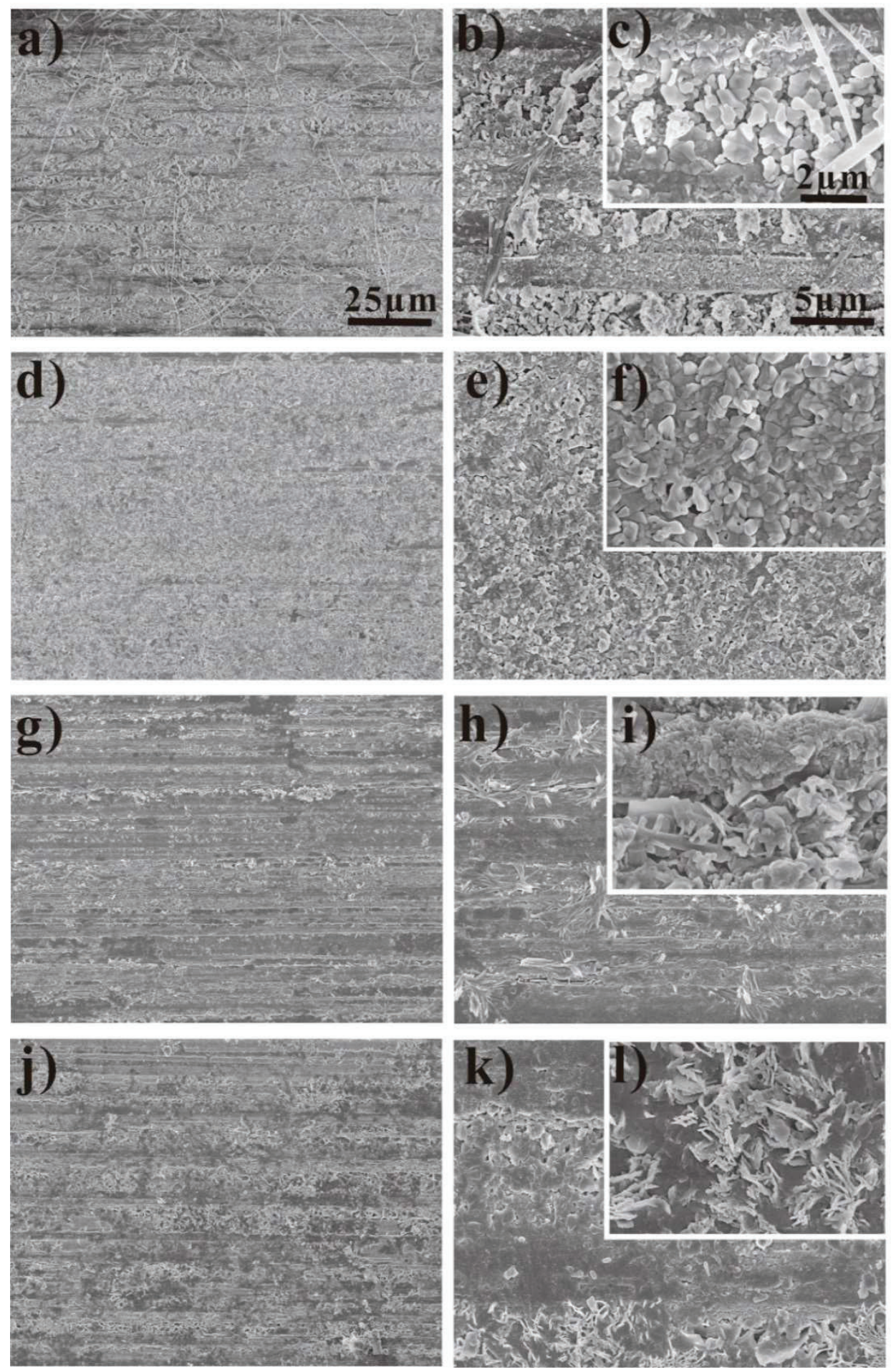

Figure 2. SEM images illustrating the surface morphologies of the MgAl-LDH layer after modification with different CIs at different magnifications. (a, b, and c) MBT@MgAl-LDH, (d, e, and f) SA@MgAl-LDH, (g, h, and i) SDBS@MgAl-LDH, and (j, k, and l) NTA@MgAl-LDH coatings. The graphs in the left and right columns and the insets have identical scale bars.

The XRD patterns and FT-IR spectra of AZ31 Mg alloy and MgAl-LDH coatings modified with different inhibitors are shown in Figure 3. Only strong characteristic peaks for Mg (JCPDS NO: 35-0821) [22] and relatively weak peaks for $\mathrm{MgO}$ and $\mathrm{Mg}(\mathrm{OH})_{2}$, which are due to the surface oxidation of $\mathrm{Mg}$, were 
detected in the pattern of the $\mathrm{Mg}$ alloy substrate (curve a1 in Figure 3a). After the deposition steps, several new peaks at $11.6^{\circ}, 20.6^{\circ}$, and $38^{\circ}$ were observed (curves a2-a6 in Figure 3a), thereby showing the layered structure characteristic of the coating and the successful growth of MgAl-LDH layer on the $\mathrm{Mg}$ alloy surface (JCPDS NO: 41-1428) [21] in accordance with theabovementioned TEM analysis. In addition, compared with the Mg alloy and bare MgAl-LDH coatings, the intensities of the feature peaks for Mg at $34.3^{\circ}$ and $36.6^{\circ}$ were evidently weakened for the CIs@MgAl-LDH coatings, thereby implying the formation of protective films related to CIs on the surface of the MgAl-LDH coatings. This inference was demonstrated by the FT-IR spectra of different MgAl-LDH coatings, as shown in Figure 3b. All spectra show characteristic peaks $\left(\mathrm{cm}^{-1}\right)$ of MgAl-LDH, namely, $3695(\mathrm{Mg}-\mathrm{OH}), 3438(\mathrm{OH}), 2922\left(\mathrm{H}_{2} \mathrm{O}-\mathrm{CO}_{3}{ }^{2-}\right)$, $1630\left(\mathrm{H}_{2} \mathrm{O}\right), 1397\left(\mathrm{CO}_{3}{ }^{2-}\right)$, and $478(\mathrm{Mg}-\mathrm{OH})$ [33], further demonstrating the formation of MgAl-LDH film on the Mg alloy substrate. In addition to these feature peaks for the MgAl-LDH layer, many new peaks appeared in the FT-IR spectra of CIs@MgAl-LDH coatings (curves b2-b5 in Figure 3b), as summarized in Table 1. Taking curve b5 as an example, two new peaks corresponding to the $\mathrm{C}-\mathrm{H}$ and $\mathrm{C}=\mathrm{C}$ stretching vibrations of thiophene ring were observed at 3084 and $1472 \mathrm{~cm}^{-1}[34,35]$. Relatively weak bands at 830 and $758 \mathrm{~cm}^{-1}$ were also identified. These bands originated from the C-S bonds in the thiophene ring of the NTA inhibitor.

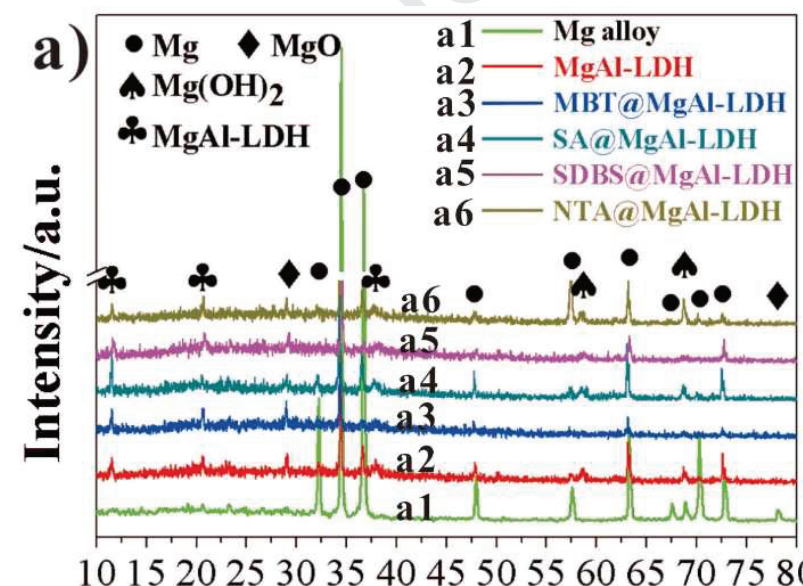

2 Theta/degree

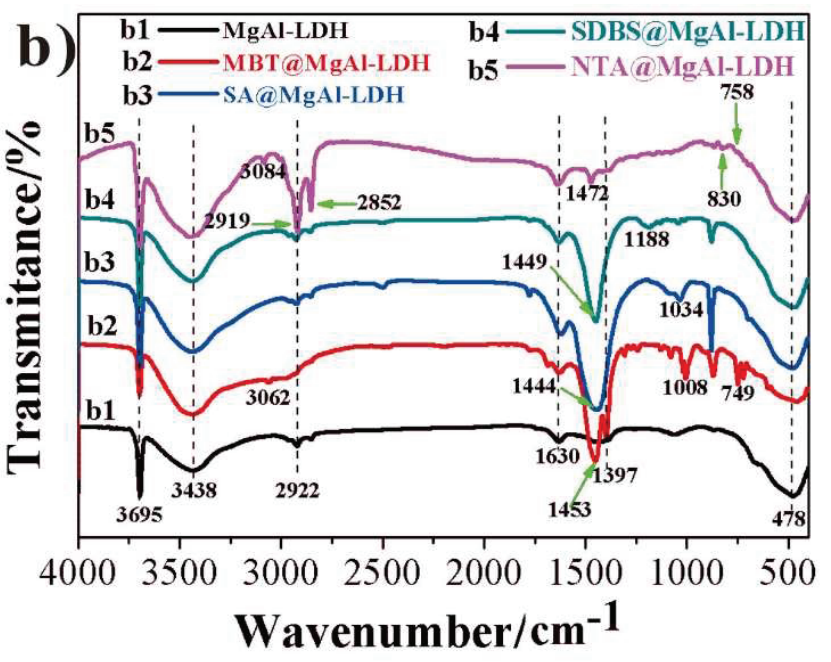

Wavenumber $/ \mathrm{cm}^{-1}$

Figure 3. (a) XRD patterns and (b) FT-IR spectra of (a1) Mg alloy, (a2, b1) MgAl-LDH, (a3, b2) MBT@MgAl-LDH, (a4, b3) SA@MgAl-LDH, (a5, b4) SDBS@MgAl-LDH, and (a6, b5) NTA@MgAl-LDH coatings. 
Table 1 Peak assignments on the FT-IR spectra of MBT@MgAl-LDH, SA@MgAl-LDH, SDBS@MgAl-LDH, and NTA@MgAl-LDH coatings.

\begin{tabular}{lcl}
\hline \multicolumn{1}{c}{ Samples } & Wavenumber $/ \mathrm{cm}^{-1}$ & \multicolumn{1}{c}{ Assignments } \\
\hline b2: MBT@MgAl-LDH [36, & 3062 & phenyl ring C-H stretching vibrations \\
37] & 1453 & phenyl ring C=C stretching vibrations \\
& 1008 & the C= $\mathrm{N}$ band intensity \\
b3: SA@MgAl-LDH [38, 39] & 749 & out-of-plane bending vibration of ArC-H bond \\
& 1444 & COO- stretching vibration \\
b4: SDBS@MgAl-LDH & 1034 & C-O stretching vibration of polysaccharide \\
[40-42] & 1449 & the C=C stretching vibrations of the benzene \\
b5: NTA@MgAl-LDH [34, & 1188 & $\mathrm{~S}=\mathrm{O}$ stretching vibration \\
35, 43] & 3084 & C- stretching vibration of the thiophene ring \\
& 2919 & C-H stretching vibration of the alkyl group \\
& 2852 & \\
& 1472 & C=C stretching vibration of the thiophene ring \\
& 830 & C-S-C stretching vibration of the thiophene ring \\
\hline
\end{tabular}

XPS was conducted to determine the chemical compositions of the CIs@MgAl-LDH films on the Mg alloy surface (Figure 4). SA consists mostly of $(1,4)$-linked $\beta$-d-mannuronic and $\alpha$-1-guluronic acid units [39], meaning $\mathrm{C}, \mathrm{H}$, and $\mathrm{O}$ elements. Compared with bare MgAl-LDH coating, new elements are not produced on the surface after SA modification, so similar XPS spectra are obtained for MgAl-LDH and SA@MgAl-LDH coatings (a1 and a3 in Figure 4a). In the spectrum of SDBS@MgAl-LDH coating, a new peak is detected clearly at $168.0 \mathrm{eV}$ which is related to sulfonate $\left(-\mathrm{SO}_{3}{ }^{-}\right)$from SDBS (a4 in Figure 4a). As for MBT@MgAl-LDH and NTA@MgAl-LDH coatings, two new peaks originating from N and S elements are observed ( $\mathrm{a} 2$ and $\mathrm{a} 5$ in Figure 4a). The peaks in the wide scan spectrum for MBT@MgAl-LDH coating are centered at 398.3 and $163.5 \mathrm{eV}$, which are assigned to N and S elements from the thiazole ring and thiol from MBT inhibitor. To gain a more accurate interpretation of the NTA adsorbed onto MgAl-LDH coating, the high-resolution XPS spectra of $\mathrm{N} \mathrm{1s,} \mathrm{S} \mathrm{2p,} \mathrm{and} \mathrm{C} 1 \mathrm{~s}$ for NTA@MgAl-LDH coating were obtained (Figure 4b-d). The spectra of N 1s and S 2p exhibit one broad symmetrical peak at 401.8 and $164.6 \mathrm{eV}$, respectively, which signifies the signal type of $\mathrm{N}$ and $\mathrm{S}$ in NTA [44]. The peak at $401.8 \mathrm{eV}$ is attributed to the quaternary ammonium salt structure, and the latter is related to the thiophene ring of as-synthesized the NTA inhibitor (Figure 4b). Notably, the binding energy of S 2p for a thiophene ring should be centered at $163.9 \mathrm{eV}[45,46]$, but an obvious positive chemical shift of 0.7 $\mathrm{eV}$ to $164.6 \mathrm{eV}$ is observed, as shown in Figure 4c. This finding indicates that electronic charges on $\mathrm{S}$ changed because of the interaction with the underlying metallic atoms from the substrate. Concerning $\mathrm{C} 1 \mathrm{~s}$, four different components can be distinguished in the deconvolution of the XPS spectrum, as presented in 
Figure 4d. The main component, located at $284.8 \mathrm{eV}$, is assigned to $\mathrm{C}-\mathrm{C} / \mathrm{C}=\mathrm{C}$ bonds originating from both NTA and ubiquitous carbon from the air [47]. The second component located at $286.0 \mathrm{eV}$ is ascribed to the C-N bond in the quaternary ammonium group of NTA [22], whereas the third and fourth components at 286.3 and $288.7 \mathrm{eV}$ are associated with C-S bonds in the thiophene ring of NTA and trace carbonyl group $(\mathrm{C}=\mathrm{O})$ from the underlying MgAl-LDH layer, respectively [44, 45].
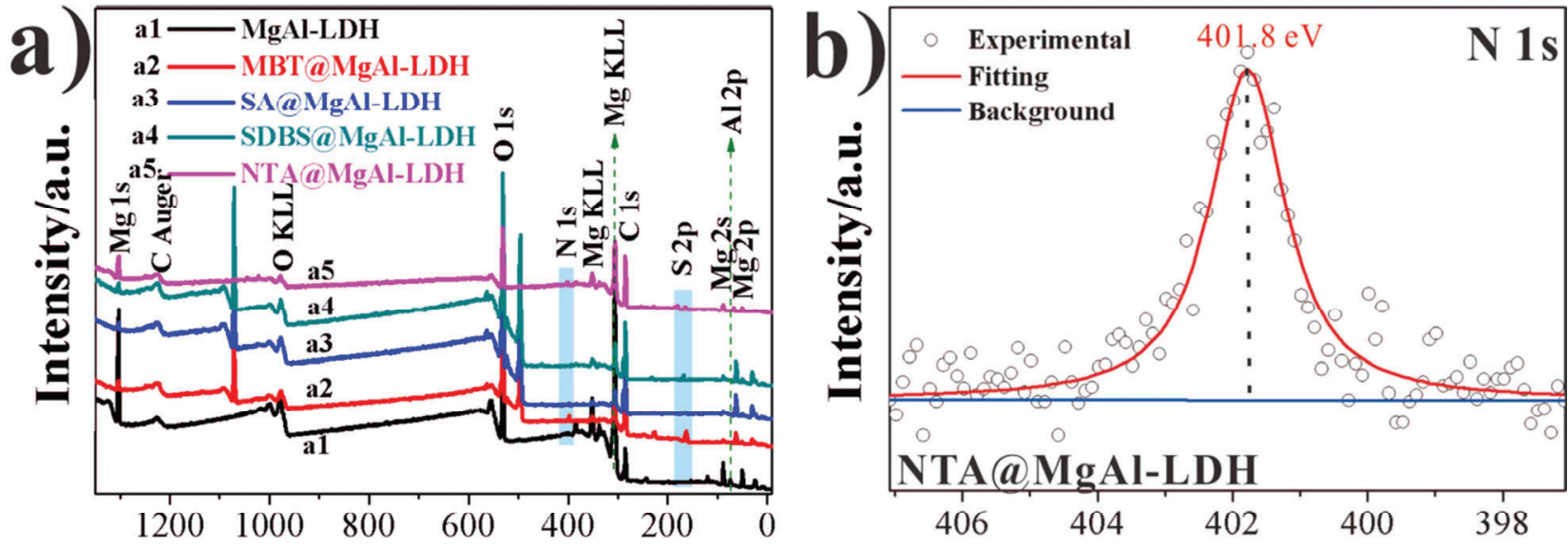

Binding Energy/eV

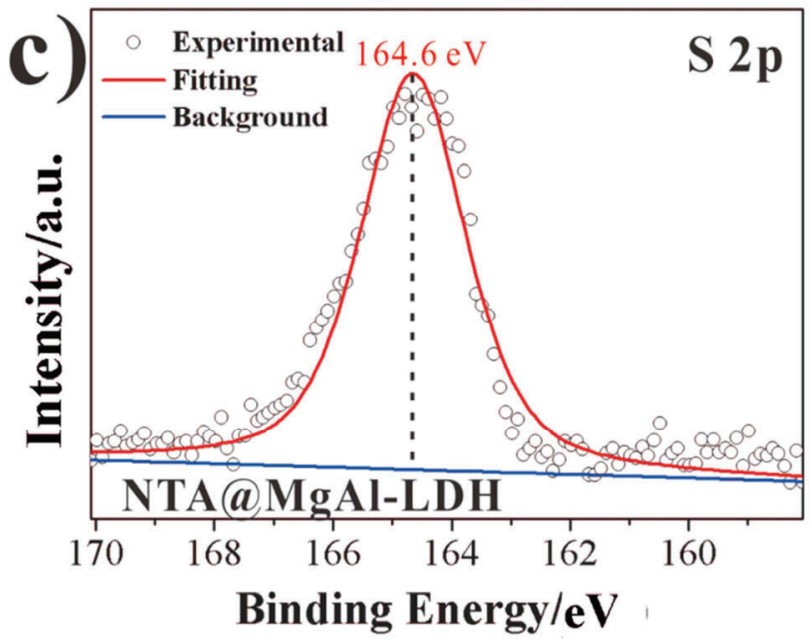

Binding Energy/eV

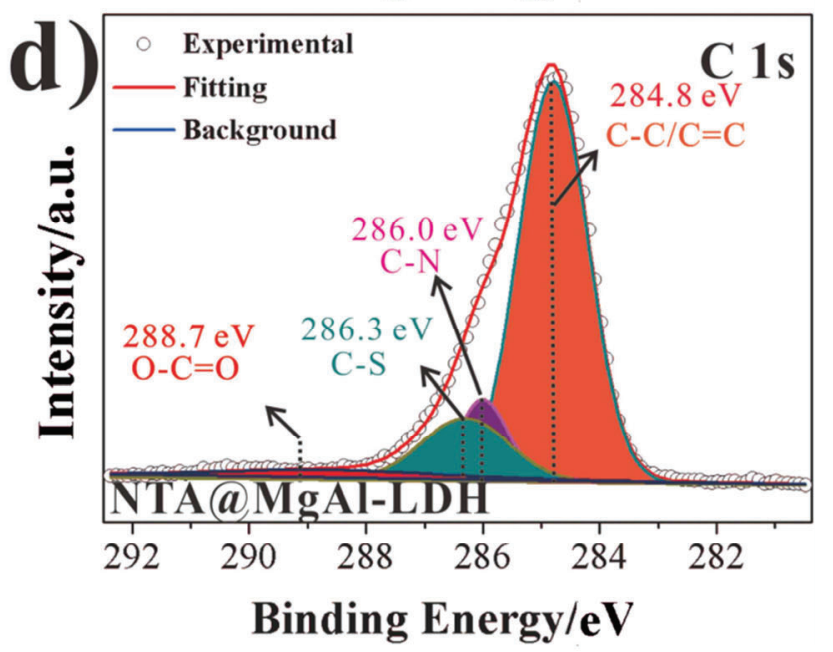

Figure 4. (a) XPS survey spectrum of the (a1) MgAl-LDH, (a2) MBT@MgAl-LDH, (a3) SA@MgAl-LDH, (a4) SDBS@MgAl-LDH and (a5) NTA@MgAl-LDH coating. The high resolution XPS spectra of (b) N 1s, (c) S 2p, and (d) C 1s for NTA@MgAl-LDH coating.

The EMPA mapping analysis of different elemental distributions on the surface of NTA@,MgAl-LDH coating is presented in Figure 5. Figures 5b-5e indicate that the sample surface is uniformly and fully covered by substances, primarily NTA inhibitor, comprising C, N, S, and O. The high content of C shown in Figure 5b is ascribed to its high content of NTA and ubiquitous $\mathrm{C}$ contamination during exposure of the sample in air, as also observed in the XPS results above. The contamination of C exists predominantly as 


\section{Journal Pre-proof}

$\mathrm{CO}_{2}$ from the air, results in a high content of $\mathrm{O}$ on the sample surface as shown in Figure 5e. $\mathrm{O}$ is unlikely from underlying MgAl-LDH depositions because $\mathrm{Mg}$ is hardly detected on the surface (Figure $\mathbf{5 f}$ ), which again demonstrates the compact and full coverage of the MgAl-LDH surface by NTA film.
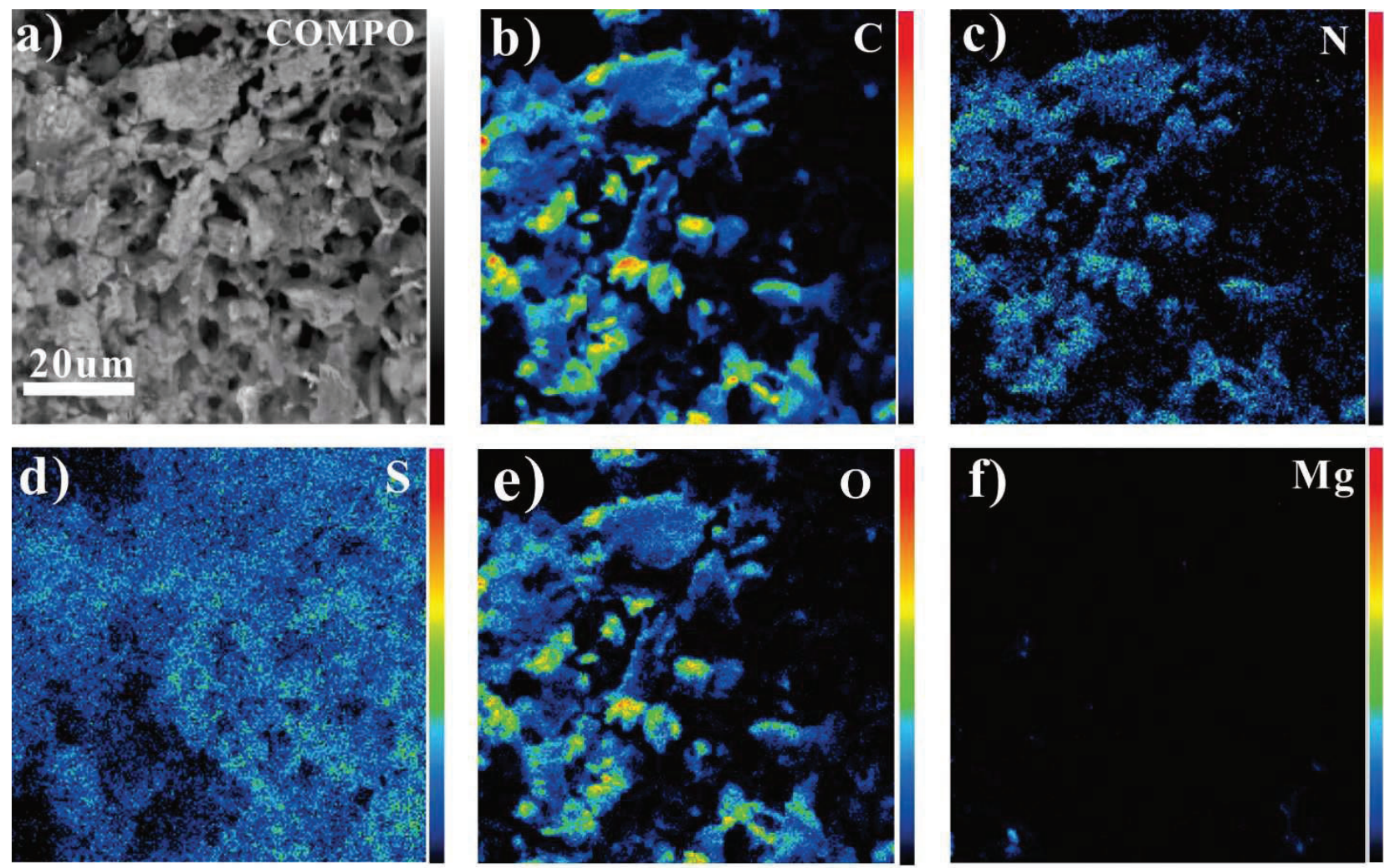

Figure 5. Results of EPMA-WDS mapping analysis showing the elemental distribution of $\mathrm{C}, \mathrm{N}, \mathrm{S}, \mathrm{O}$, and $\mathrm{Mg}$ on the surface of NTA@MgAl-LDH coating.

Electrochemical tests, including EIS and potentiodynamic polarization curves, were obtained to estimate the corrosion resistance of the different samples [48-51]. Generally, a higher value of impedance modulus at lower frequency means greater improvement in the corrosion resistance of the coating [21, 22, 52, 53]. Obviously, the MgAl-LDH coating shows a much higher modulus at low frequencies thaan $\mathrm{Mg}$ alloy (Figure 6a). After modification of the MgAl-LDH with CIs, the moduli of all CIs@MgAl-LDH coatings further increase evidently. For example, considering the moduli at $0.1 \mathrm{~Hz}\left(|\mathrm{Z}|_{f=0.1 \mathrm{~Hz}}\right)$, the $|\mathrm{Z}|_{f=0.1}$ $\mathrm{Hz}$ for $\mathrm{Mg}$ alloy substrate is about $1000 \Omega \mathrm{cm}^{2}$. This value increases to $15.6 \mathrm{M} \Omega \mathrm{cm}^{2}$ for $\mathrm{MgAl}-\mathrm{LDH}$ coating, and further increases after CIs treatment until they exceed $67.9 \mathrm{M} \Omega \mathrm{cm}^{2}$. The enhanced corrosion resistance of the coatings is also distinguished clearly by the Bode phase plots, as shown in Figure $6 \mathrm{~b}$ and Nyquist graphs as shown in Figure 6c-f. In the case of uncoated Mg alloy substrate in Figure 6b, the oxide 
film formed by the natural oxidation of $\mathrm{Mg}$ is porous and incompact [8], showing very limited coating resistance. Thus, the alternate current at high frequency is prone to go through a resistor and the phase angle is almost $0^{\circ}[54]$. As for the MgAl-LDH and CIs@MgAl-LDH coatings in Figure 6b, they are complete and have good barrier characteristics, indicating that alternate current at high frequency tends to go through a capacitor and the phase angles approach $-90^{\circ}[53,54]$. In particular, the phase angle of NTA@MgAl-LDH coating at $100 \mathrm{kHz}$ is $-89.24^{\circ}$, exhibiting the intact property of the coating and excellent corrosion protection. The diameter of the capacitive loop in a Nyquist graph is proportional to the corrosion resistance of the coating. The Nyquist plots in Figure 6c clearly show that NTA@MgAl-LDH coating has the largest diameter in the capacitive loops, manifesting the best corrosion protection of the coating among all samples. The diameter of the loop for CIs@MgAl-LDH coatings are so large that the plots for bare MgAl-LDH coating and $\mathrm{Mg}$ alloy are hard to distinguish and totally overwhelmed, respectively, at the same scale of the x-axis in a Nyquist graph. Accordingly, the Nyquist plots are presented using different scales in x-axis, as shown in Figure 6d-f. To further elucidate the EIS spectra, electrical equivalent circuits (ECs) were used to fit the experimental impedance spectra with ZSimpWin software. The EC depicted in Figure 6g is used to fit the EIS spectrum of bare Mg alloy and includes $R_{\mathrm{s}}$ for solution resistance and $R_{\mathrm{ct}}$ for charge transfer resistance. Constant phase element (CPE), which is related to the capacitance of electrical double layer $\left(C P E_{\mathrm{dl}}\right)$, is used to replace the pure capacitor to increase fitting accuracy. $L$ stands for inductance and stands $R_{\mathrm{L}}$ for the resistance of induction associated with $\mathrm{Mg}$ oxidation and the adsorption/desorption of corrosion products at vulnerable sites [52]. In addition to some of the abovementioned electrochemical parameters, the EC of bare MgAl-LDH coating as presented in Figure $6 \mathrm{~h}$ includes $R_{1}$ and $\mathrm{CPE}_{1}$, which are related to the resistance and capacitance of MgAl-LDH film, respectively. $Z_{\mathrm{W}}$ stands for diffusion resistance related to ion exchangeability [22]. After coverage of the MgAl-LDH coating by formation of an inhibitor film, a new EC without $Z_{\mathrm{W}}$ but with a three-time constant is adopted (Figure 6i). The new electrochemical parameters include $R_{2}$ and $C P E_{2}$ for resistance and capacitance of inhibitor film, respectively. The fitting values of the electrochemical parameters based on the ECs above are listed in Table 2. $R_{1}$ and $R_{\mathrm{ct}}$ for all coatings become very high, especially for CIs@MgAl-LDH coatings, which increase more significantly than bare MgAl-LDH coating 
because of the protection of inhibitor films. For instance, $R_{1}$ and $R_{\mathrm{ct}}$ for bare MgAl-LDH coating are 1.030 and 22.16 M $\Omega \mathrm{cm}^{2}$, respectively, whereas those for NTA@MgAl-LDH coating increase by near 600 times to $614.2 \mathrm{M} \Omega \mathrm{cm}^{2}$ and more than 80 times to $1837 \mathrm{M} \Omega \mathrm{cm}^{2}$, respectively. In summary, the corrosion protection of bare MgAl-LDH coating to $\mathrm{Mg}$ alloy is good and can be enhanced remarkably after modification by different CIs, among which the inhibitor synthesized in this work, i.e., NTA, shows the best effectiveness.
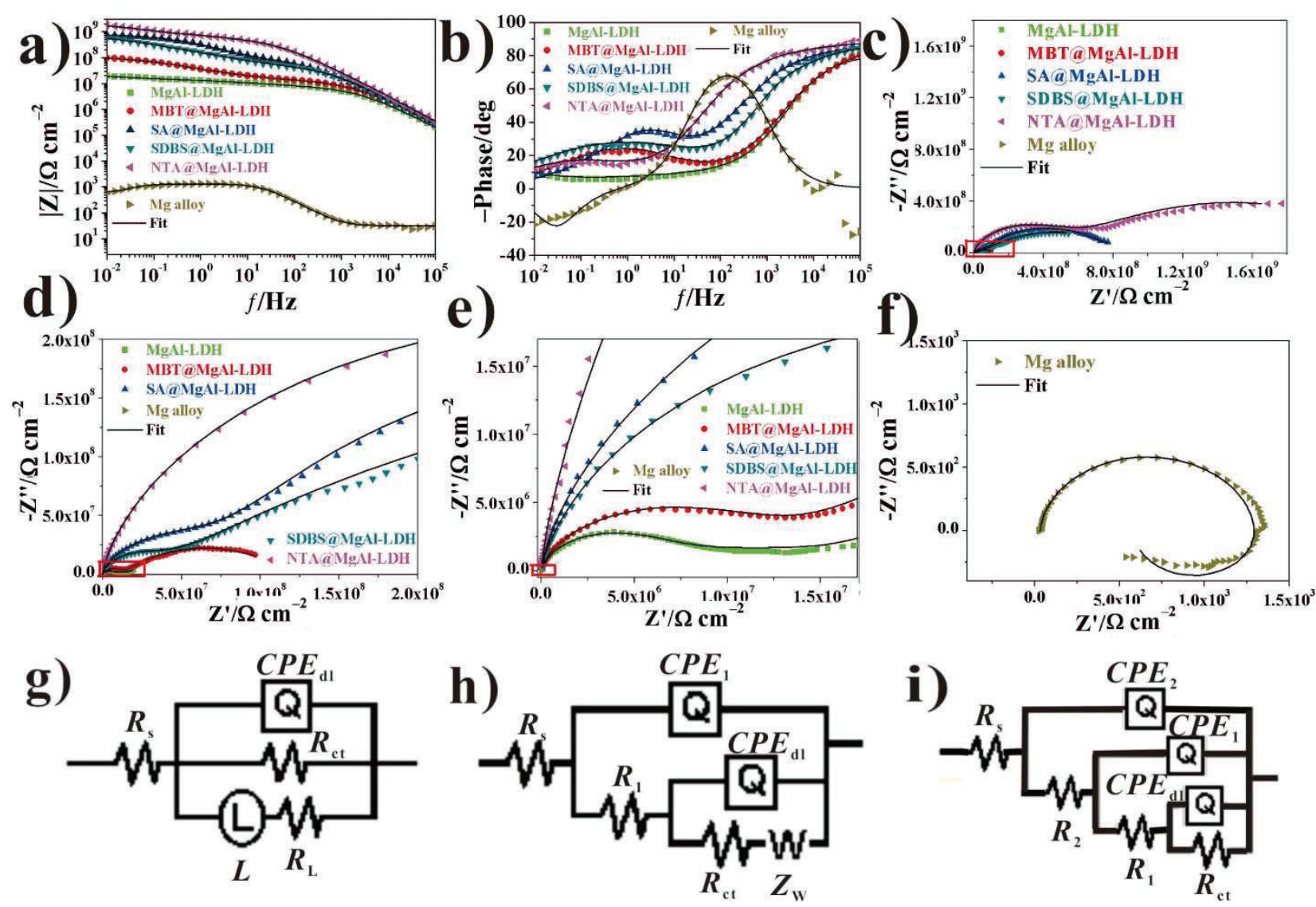

Figure 6. EIS diagrams of $\mathrm{Mg}$ alloy, MgAl-LDH, and CIs@MgAl-LDH coatings in 3.5 wt.\% NaCl solution. (a, b) Bode magnitude and Bode phase plots. (c) Nyquist plots. (d) Magnified Nyquist plots for the square area in (c). (e) Magnified Nyquist plots for the square area in (d). (f) Nyquist plots for AZ31 Mg alloy substrate. The dotted and solid lines are experimental and fitting results, respectively. Equivalent circuits of (g) AZ31 Mg alloy substrate, (h) MgAl-LDH coating, and (i) CIs@MgAl-LDH coatings. 
Table 2 Fitting electrochemical parameters for EIS spectra of different MgAl-LDH coatings

\begin{tabular}{|c|c|c|c|c|c|c|c|c|c|c|}
\hline Coatings & $\begin{array}{c}R_{\mathrm{s}} / \\
\Omega \mathrm{cm}^{2}\end{array}$ & $\begin{array}{c}Y_{2} / 10^{-12} \\
\mathrm{~S} \mathrm{~s}^{\mathrm{n}} \mathrm{cm}^{-2}\end{array}$ & $\begin{array}{l}R_{2} / \\
\mathrm{M} \Omega \\
\mathrm{cm}^{2} \\
\end{array}$ & $\begin{array}{c}Y_{1} / 10^{-11} \\
\mathrm{~S} \mathrm{~s}^{\mathrm{n}} \mathrm{cm}^{-2}\end{array}$ & $\begin{array}{c}R_{1} / \\
\mathrm{M} \Omega \mathrm{cm}^{2}\end{array}$ & $\begin{array}{c}Y_{\mathrm{dl}} / 10^{-8} \\
\mathrm{~S} \mathrm{~s}^{\mathrm{n}} \mathrm{cm}^{-2}\end{array}$ & $\begin{array}{l}R_{\mathrm{ct}} / \\
\mathrm{M} \Omega \\
\mathrm{cm}^{2} \\
\end{array}$ & $\begin{array}{c}Z_{\mathrm{W}} / 10^{-7} \\
\mathrm{~S} \mathrm{~s}^{0.5} \\
\mathrm{~cm}^{-2}\end{array}$ & $\begin{array}{c}|\mathrm{Z}|_{f=0.1 \mathrm{~Hz}} / \\
\mathrm{M} \Omega \mathrm{cm}^{2}\end{array}$ & $\begin{array}{c}\chi^{2} / \\
10^{-4}\end{array}$ \\
\hline MgAl-LDH & 4 & N/A & $\mathrm{N} / \mathrm{A}$ & 2.862 & 1.030 & 2.808 & 22.16 & 2.815 & 15.63 & 9.07 \\
\hline $\begin{array}{c}\text { MBT@ } \\
\text { MgAl-LDH }\end{array}$ & 5 & 7.735 & 1.109 & 0.5804 & 13.55 & 1.259 & 102.6 & N/A & 67.94 & 3.59 \\
\hline$\frac{\text { SA@ }}{\text { MgAl-LDH }}$ & 4 & 1.112 & 4.939 & 0.6049 & 142.6 & 0.03766 & 713.1 & $\mathrm{~N} / \mathrm{A}$ & 592.9 & 2.15 \\
\hline $\begin{array}{c}\text { SDBS@ } \\
\text { MgAl-LDH }\end{array}$ & 4.5 & 8.519 & 7.573 & 5.315 & 29.14 & 0.3077 & 883.5 & N/A & 315.9 & 3.76 \\
\hline $\begin{array}{c}\text { NTA@ } \\
\text { MgAl-LDH }\end{array}$ & 6.5 & 5.531 & 7.910 & 6.925 & 614.2 & 0.1575 & 1837 & N/A & 1097.7 & 6.62 \\
\hline
\end{tabular}

Figure 7 presents the potentiodynamic polarization curves of bare $\mathrm{Mg}$ alloy and different coatings in $\mathrm{NaCl}$ solution at $298 \mathrm{~K}$. Given that $\mathrm{Mg}$ alloy has a negative difference effect (NDE) in the anodic branch, the corrosion current density $\left(j_{\text {corr }}\right)$ of bare sample was obtained by only cathodic Tafel extrapolation [55, 56]. Figure 7 shows that compared with uncoated $\mathrm{Mg}$ alloy, the corrosion potentials $\left(E_{\text {corr }}\right)$ and $j_{\text {corr }}$ of the coatings shift obviously toward more positive and lower values, respectively, indicating the significantly enhanced corrosion resistance in neutral corrosive environment in terms of both thermodynamics and kinetics. According to the electrochemical parameters listed in Table 3 based on the potentiodynamic polarization curves, the $E_{\text {corr }}$ of substrate is $-1.5 \mathrm{~V}$, which shifts positively by $0.75 \mathrm{~V}$ to $-0.75 \mathrm{~V}$ after coating with MgAl-LDH film. Further shifts to around $-0.65 \mathrm{~V}$ are observed after being impregnated with CIs, except for NTA inhibitor that results in the largest $E_{\text {corr }}$ shift to $-0.437 \mathrm{~V}$, exhibiting the greatest improvement in corrosion protection. The $j_{\text {corr }}$ of Mg alloy is $5.888 \mu \mathrm{A} \mathrm{cm}^{-2}$, which decreases by three orders of magnitude to $1.543 \mathrm{nA} \mathrm{cm}{ }^{-2}$ for MgAl-LDH coating. After CI treatment of the coatings, $j_{\text {corr }}$ is further reduced by $1 / 4$ to 0.3976 nA cm ${ }^{-2}$ for MBT@MgAl-LDH coating, by about 1/25 for both SA@MgAl-LDH and MBT@MgAl-LDH coatings, and by almost $1 / 40$ to 0.04001 nA cm ${ }^{-2}$ for NTA@MgAl-LDH coating. The variations in $E_{\text {corr }}$ and $j_{\text {corr }}$ show that the corrosion protection of the different coatings decreases in the following order: NTA@MgAl-LDH > SDBS@MgAl-LDH $\approx$ SA@MgAl-LDH > MBT@MgAl-LDH > MgAl-LDH coatings. This finding well agrees with the EIS results. 


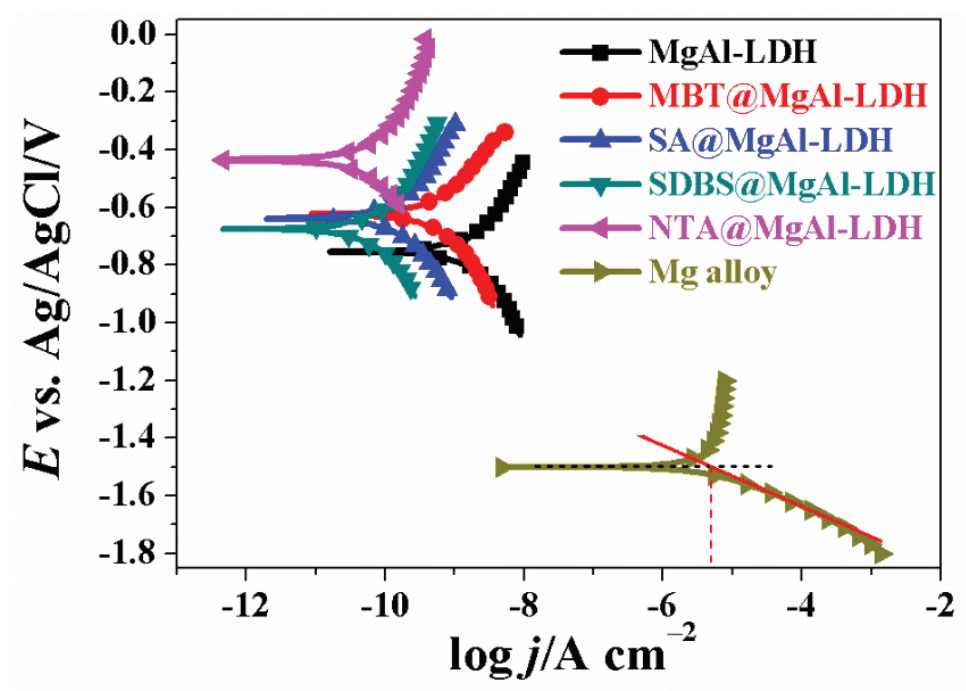

Figure 7. Potentiodynamic polarization curves of Mg alloy, MgAl-LDH and different CIs@MgAl-LDH coatings in 3.5 wt. $\% \mathrm{NaCl}$ medium.

Table 3 Electrochemical parameters based on the potentiodynamic polarization curves

\begin{tabular}{ccccc}
\hline Samples & $\beta_{\mathrm{a}} / \mathrm{mV} \mathrm{dec}^{-1}$ & $-\beta_{\mathrm{c}} / \mathrm{mV} \mathrm{dec}^{-1}$ & $E_{\mathrm{corr}} / \mathrm{V}$ & $j_{\mathrm{corr}} / \mathrm{nA} \mathrm{cm}^{-2}$ \\
\hline Mg alloy & $/$ & 112 & -1.50 & 5888 \\
MgAl-LDH & 205 & 196 & -0.750 & 1.543 \\
MBT@MgAl-LDH & 198 & 199 & -0.623 & 0.3976 \\
SA@MgAl-LDH & 207 & 206 & -0.658 & 0.06215 \\
SDBS@MgAl-LDH & 187 & 210 & -0.674 & 0.06141 \\
NTA@MgAl-LDH & 194 & 190 & -0.437 & 0.04001 \\
\hline
\end{tabular}

To observe the corrosion protection capability and durability of the NTA@MgAl-LDH coating when defects occur, an artificial scratch was made, and immersion tests were carried out. Results are shown in Figure 8. Before soaking a sample, it was scratched with a knife to form a cut with the same depth and width across both NTA-treated and NTA-free areas (Figure 8a-c). After $8 \mathrm{~h}$ of immersion in $\mathrm{NaCl}$ solution, both cuts in NTA-treated and NTA-free areas are filled to some extent. Compared with the cut on the NTA-free side, the precipitations in the cut on the NTA-treated side is more compact with fewer and smaller cracks, although the former shows more complete filling. This result is attributed to the different chemical compositions of the depositions resulting from different self-healing reactions. First, $\mathrm{CO}_{3}{ }^{2-}$ in LDH coating can be released by exchanging with $\mathrm{Cl}^{-}$as expressed in Equation (1) to form $\mathrm{MgCO}_{3}$ precipitate (Equation (2)) when the sample is soaked in $\mathrm{NaCl}$ solution, although usually the affinity of $\mathrm{CO}_{3}{ }^{2-}$ for $\mathrm{LDH}$ intercalation is higher than that of $\mathrm{Cl}^{-}$anion $[22,57]$. The $\mathrm{MgCO}_{3}$ precipitate can further 
react with hydroxyl ions to form a more stable $\mathrm{Mg}(\mathrm{OH})_{2}$ deposition, as depicted in Equation (3), because the latter has a smaller solubility product constant $\left(K_{\mathrm{sp}}=5.610^{-12}\right)$ than $\mathrm{MgCO}_{3}\left(K_{\mathrm{sp}}=6.810^{-6}\right)$ at $25^{\circ} \mathrm{C}$ [22]. $\mathrm{Mg}(\mathrm{OH})_{2}$ deposition acts as a protective film, covering the vulnerable sites and repairing the physical defects to some extent, accounting for the formation of precipitation in the cut and good corrosion protection for bare MgAl-LDH and NTA@MgAl-LDH coatings.

$$
\begin{gathered}
\mathrm{LDH}-\mathrm{CO}_{3}^{2-}+2 \mathrm{Cl}^{-} \rightarrow \mathrm{LDH}-2 \mathrm{Cl}^{-}+\mathrm{CO}_{3}^{2-} \\
\mathrm{Mg}^{2+}+\mathrm{CO}_{3}^{2-} \rightarrow \mathrm{MgCO}_{3} \downarrow \\
\mathrm{MgCO}_{3}+2 \mathrm{OH}^{-} \rightarrow \mathrm{Mg}(\mathrm{OH})_{2} \downarrow+\mathrm{CO}_{3}^{2-}
\end{gathered}
$$

However, considering that the cut into the matrix is sufficiently deep, the exposed Mg alloy corrodes and the exposed area enlarges soon if the self-healing process cannot be triggered immediately and if the vulnerable sites cannot be filled enough by the compact and stable precipitations. In addition to the ion exchange process, the NTA near the scratch can move to the exposed sites to form an adsorbed protective film when defects were produced in the NTA@MgAl-LDH coating, as supported by subsequent EDS analysis (Figure 8j-k) and DFT calculations. Thus, the cut in this case can be protected better than that without NTA. Figure 8j clearly shows that the depositions in the cut on the left side contain N and S originating from NTA, wherreas these elements cannot be detected in the cut on the right side. We deduce that the substances in the cut on the right side include more porous corrosion products resulting from more severe corrosion at the initial stage of matrix exposure, accounting for the larger amount of depositions, cracks, and cut after $8 \mathrm{~h}$ of immersion than in the left case (Figure 8e). Remarkably, after $48 \mathrm{~h}$ of immersion, although some cracks can be found at a high magnification for the left cut (Figure 8g), the depositions are much more compact than that in the right cut (Figure 8h) that shows many big crevices. These large crevices are difficult to provide good corrosion protection to the Mg alloy during long-term immersion in corrosive environments. 


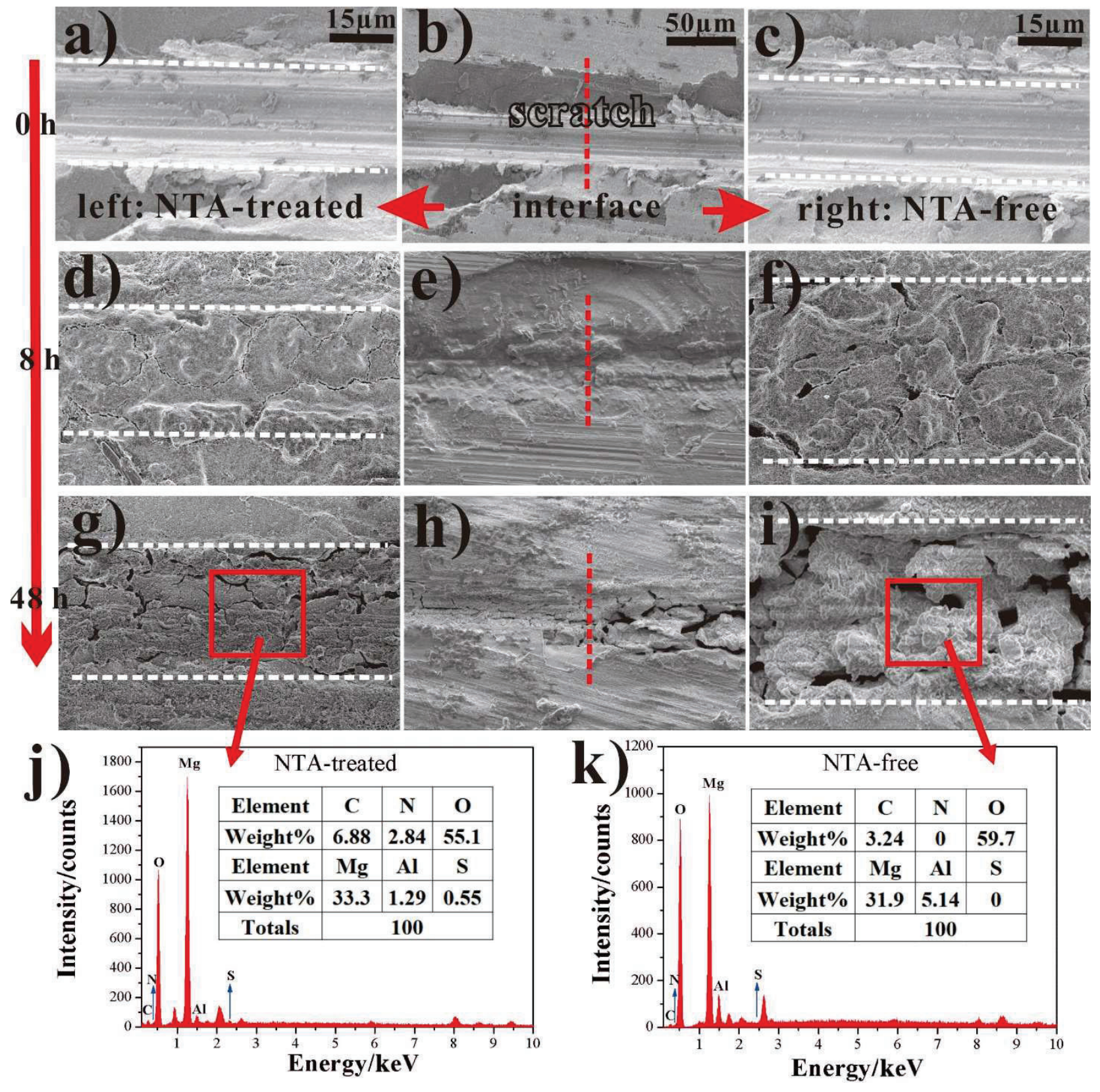

Figure 8. SEM images showing the morphological evolutions of MgAl-LDH coating with and without modification by NTA after artificial scratch and soaking in $3.5 \mathrm{wt} . \% \mathrm{NaCl}$ solution. (a, b, c) Fresh sample after scratch, (d, e, f) 8 h, and $(\mathrm{g}, \mathrm{h}, \mathrm{f}) 48 \mathrm{~h}$ of immersion. The graphs in the left column shows the area treated with NTA $(\mathrm{a}, \mathrm{d}, \mathrm{g})$, the middle column is the interface including both NTA-treated and NTA-free areas (b,e, h); those in the right column is the area without NTA treatment (c, f, i). Middle column: 500 times; left and right column: 1500 times. EDS of NTA-treated (j) and NTA-free $(\mathrm{k})$ areas of MgAl-LDH coating after $48 \mathrm{~h}$ of immersion.

DFT calculations have been used to study the properties of $\mathrm{CI} /$ surface mechanisms and describe the structural nature of CIs during corrosion [31, 32]. To demonstrate the assumption of NTA movement from near the cut to the exposed sites during the self-repairing process, DFT calculations were performed to optimize the geometric and electronic structures of NTA and the possible complexes, as well as to calculate $E_{\mathrm{a}}$ to explore the interactions between NTA molecule and $\mathrm{Mg}(0001)$ surface. $E_{\mathrm{a}}$ was calculated based on 
the energy difference of the system after and before adsorption, i.e., $E_{\text {ad }}=E[\mathrm{NTA}-\mathrm{Mg}(0001)$ surface $]-$ $E[\mathrm{NTA}]-E[\mathrm{Mg}(0001)$ surface], where $E[\mathrm{NTA}-\mathrm{Mg}(0001)$ surface $], E[\mathrm{NTA}]$, and $E[\mathrm{Mg}(0001)$ surface $]$ represent the energies of NTA molecule adsorbed onto the Mg surface, the energy of an isolated NTA molecule, and the energy of clean Mg (0001) surface, respectively. DFT-D3 method was used to account for the van der Waals interactions between NTA molecule and $\mathrm{Mg}$ surface. $E_{\text {ad }}$ is found to be $-1.91 \mathrm{eV}$ $\left(-184.2 \mathrm{~kJ} \mathrm{~mol}^{-1}\right)$, indicating a chemisorption process, in which NTA adsorbs both chemically and physically onto $\mathrm{Mg}$ surface with a stable state. According to recent studies, the obtained $E_{\text {ad }}$ in the present system is lower than the $E_{\text {ad }}$ of $* \mathrm{OH}$ and $\mathrm{H}_{2} \mathrm{O}$ molecules on $\mathrm{Mg}(0001), \mathrm{MgO}(0001)$, and $\mathrm{Mg}(\mathrm{OH})_{2}(0001)$ [31]. Thus, the favorability of adsorption of NTA on the surfaces of exposed Mg and subsequently produced $\mathrm{Mg}(\mathrm{OH})_{2}$ precipitation film benefits the filling of big cracks and pores in the $\mathrm{MgO}$ and $\mathrm{Mg}(\mathrm{OH})_{2}$ precipitates to form a more compact composite film repair the physical defects, and enhance the corrosion protection ability., As shown in Figure 9, the optimized structure of NTA on Mg (0001) surface indicates that the thiophene ring is almost parallel to the $\mathrm{Mg}(0001)$ crystal plane. This spatial arrangement of atoms favors the coordination of conjugated $\pi$-bonds in NTA and empty orbit in Mg atoms, proving that the NTA molecules can be chemisorbed onto the $\mathrm{Mg}$ crystal face. The chemical adsorption between $\mathrm{Br}^{-}$and $\mathrm{Mg}$ strengthens the electrostatic interaction between $\mathrm{N}^{+}, \mathrm{Br}^{-}$and $\mathrm{Mg}$ in the adsorption system, thereby improving the physicochemical adsorption capacity of NTA.

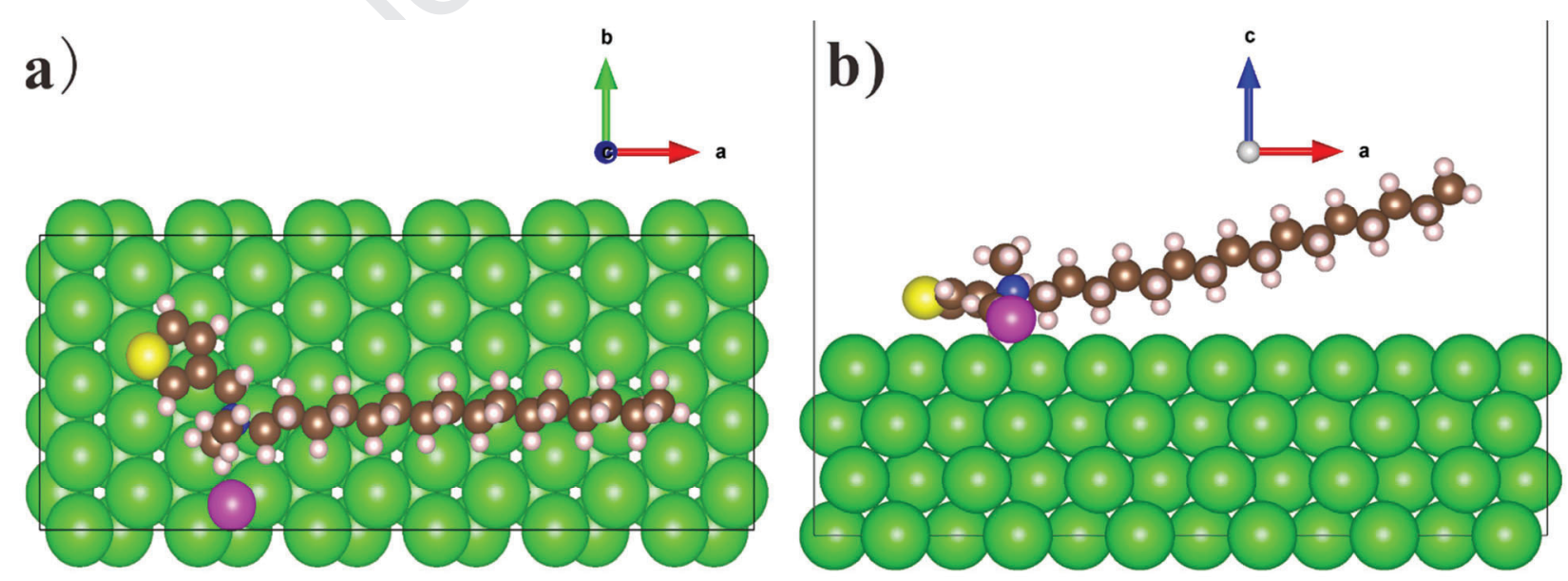

Figure 9. (a) Top and (b) side views of computational models for the optimized adsorption structure of NTA molecule onto the Mg (0001) surface with four layers. The green, brown, white, blue, yellow and magenta balls stand for Mg, $\mathrm{C}, \mathrm{H}, \mathrm{N}, \mathrm{S}$ and $\mathrm{Br}$ atoms, respectively. 


\section{CONCLUSION}

Composite coatings consisting of underlying MgAl-LDH layer and outer films of different CIs were fabricated on AZ31 Mg alloy by a hydrothermal method followed through a simple immersion step. Compared with bare MgAl-LDH film, the composite coatings exhibit higher compactness and significant enhancement in corrosion resistance in $\mathrm{NaCl}$ solution. Most importantly, we demonstrate that the as-synthesized thiophene derivative inhibitor (i.e., NTA) shows the best effectiveness in enhancing the corrosion protection of MgAl-LDH film among all commercial CIs that are usually reported in $\mathrm{LDH}$ coating studies. In addition to the strengthened physical barrier, the exceptional anticorrosion capability of NTA@MgAl-LDH coating may be attributed to the self-healing process to repair the physical damage through the formation of new compact barrier films by the (1) chemical reactions between $\mathrm{Mg}^{2+}$ ions and $\mathrm{CO}_{3}{ }^{2-}$ originating from the LDH layer, and (2) physicochemical adsorption of NTA onto the $\mathrm{Mg}(0001)$ crystal plane and oxide of $\mathrm{Mg}$ as supported by DFT calculations. All these results provide new insights into the interactions of NTA with MgAl-LDH depositions and Mg alloy substrate and can serve as guidelines in designing thiophene-contained CIs for the modification of MgAl-LDH primer and thus achieve superior corrosion protection.

\section{ASSOCIATED CONTENT}

\section{Supporting Information}

${ }^{1} \mathrm{H}$ NMR, ${ }^{13} \mathrm{C}$ NMR, GCMS and FT-IR spectra (raw data), tables based on these raw data and a brief description to demonstrate the chemical formula and structure of NTA.

\section{AUTHOR INFORMATION}

ORCID: Zhi-Hui Xie: 0000-0002-1556-0700

\section{ACKNOWLEDGMENTS}

This work has been supported by the National Natural Science Foundation of China (NSFC, 51501157); Young Elite Scientists Sponsorship Program by China Association for Science and Technology (YESS, 2018QNRC001), and Sichuan Science and Technology Program (2018JY0483). CF would like to express his gratitude to PALS for its support. 


\section{REFERENCE}

[1] Z. Li, W. Yang, Q. Yu, Y. Wu, D. Wang, J. Liang, F. Zhou, New method for the corrosion resistance of AZ31 Mg alloy with a porous micro-arc oxidation membrane as an ionic corrosion inhibitor container, Langmuir, 35 (2019) 1134-1145.

[2] P. Xiong, Z. Jia, M. Li, W. Zhou, J. Yan, Y. Wu, Y. Cheng, Y. Zheng, Biomimetic Ca, Sr/P-doped silk fibroin films on $\mathrm{Mg}-1 \mathrm{Ca}$ alloy with dramatic corrosion resistance and osteogenic activities, ACS Biomater. Sci. Eng., 4 (2018) 3163-3176.

[3] J. Dou, Y. Chen, Y. Chi, H. Li, G. Gu, C. Chen, Preparation and characterization of a calcium-phosphate-silicon coating on a Mg-Zn-Ca alloy via two-step micro-arc oxidation, Phys. Chem. Chem. Phys., 19 (2017) 15110-15119. [4] R.L. Liu, J.R. Scully, G. Williams, N. Birbilis, Reducing the corrosion rate of magnesium via microalloying additions of group 14 and 15 elements, Electrochim. Acta, 260 (2018) 184-195.

[5] M.P. Brady, A.V. Ievlev, M. Fayek, D.N. Leonard, M.G. Frith, H.M. Meyer, A.J. Ramirez-Cuesta, L.L. Daemen, Y. Cheng, W. Guo, J.D. Poplawsky, O.S. Ovchinnikova, J. Thomson, L.M. Anovitz, G. Rother, D. Shin, G.-L. Song, B. Davis, Rapid diffusion and nanosegregation of hydrogen in magnesium alloys from exposure to water, ACS Appl. Mater. Interfaces, 9 (2017) 38125-38134.

[6] C.Q. Li, D.K. Xu, X.B. Chen, B.J. Wang, R.Z. Wu, E.H. Han, N. Birbilis, Composition and microstructure dependent corrosion behaviour of Mg-Li alloys, Electrochim. Acta, 260 (2018) 55-64.

[7] Y. Zhang, P. Gore, W. Rong, Y. Wu, Y. Yan, R. Zhang, L. Peng, J.-F. Nie, N. Birbilis, Quasi-in-situ STEM-EDS insight into the role of $\mathrm{Ag}$ in the corrosion behaviour of Mg-Gd-Zr alloys, Corros. Sci., 136 (2018) 106-118.

[8] Z.-H. Xie, D. Li, Z. Skeete, A. Sharma, C.-J. Zhong, Nanocontainer-enhanced self-healing for corrosion-resistant Ni coating on Mg alloy, ACS Appl. Mater. Interfaces, 9 (2017) 36247-36260.

[9] G. Duan, L. Yang, S. Liao, C. Zhang, X. Lu, Y. Yang, B. Zhang, Y. Wei, T. Zhang, B. Yu, X. Zhang, F. Wang, Designing for the chemical conversion coating with high corrosion resistance and low electrical contact resistance on AZ91D magnesium alloy, Corros. Sci., 135 (2018) 197-206.

[10] Z. Rajabalizadeh, D. Seifzadeh, Application of electroless Ni-P coating on magnesium alloy via $\mathrm{CrO}_{3} / \mathrm{HF}$ free titanate pretreatment, Appl. Surf. Sci., 422 (2017) 696-709.

[11] D. Jiang, X. Xia, J. Hou, X. Zhang, Z. Dong, Enhanced corrosion barrier of microarc-oxidized Mg alloy by self-healing superhydrophobic silica coating, Ind. Eng. Chem. Res., 58 (2019) 165-178.

[12] X. Lu, C. Blawert, Y. Huang, H. Ovri, M.L. Zheludkevich, K.U. Kainer, Plasma electrolytic oxidation coatings on $\mathrm{Mg}$ alloy with addition of $\mathrm{SiO}_{2}$ particles, Electrochim. Acta, 187 (2016) 20-33.

[13] D. Li, F. Chen, Z.-H. Xie, S. Shan, C.-J. Zhong, Enhancing structure integrity and corrosion resistance of Mg alloy by a two-step deposition to avoid F ions etching to nano- $\mathrm{SiO}_{2}$ reinforcement, J. Alloys Compd., 705 (2017) $70-78$.

[14] Z.H. Xie, F. Chen, S. Xiang, J. Zhou, Z. Song, G. Yu, Studies of several pickling and activation processes for electroless Ni-P plating on AZ31 magnesium alloy, J. Electrochem. Soc., 162 (2015) D115-D123.

[15] D. Yan, G. Yu, B. Hu, J. Zhang, Z. Song, X. Zhang, An innovative procedure of electroless nickel plating in fluoride-free bath used for AZ91D magnesium alloy, J. Alloys Compd., 653 (2015) 271-278.

[16] P. Shoghi, D. Seifzadeh, M. Gholizadeh-Gheshlaghi, A. Habibi-Yangjeh, Pretreatment-free Ni-P plating on magnesium alloy at low temperatures, Trans. Nonferrous Met. Soc. China, 28 (2018) 2478-2488.

[17] S. Nezamdoust, D. Seifzadeh, Application of CeH-V/ sol-gel composite coating for corrosion protection of AM60B magnesium alloy, Trans. Nonferrous Met. Soc. China, 27 (2017) 352-362.

[18] S. Nezamdoust, D. Seifzadeh, Z. Rajabalizadeh, PTMS/OH-MWCNT sol-gel nanocomposite for corrosion protection of magnesium alloy, Surf. Coat. Technol., 335 (2018) 228-240.

[19] L. Yan, M. Zhou, X. Pang, K. Gao, One-step in situ synthesis of reduced graphene oxide/Zn-Al layered double 
hydroxide film for enhanced corrosion protection of magnesium alloys, Langmuir, 35 (2019) 6312-6320.

[20] Y. Cao, D. Zheng, X. Li, J. Lin, C. Wang, S. Dong, C. Lin, Enhanced corrosion resistance of superhydrophobic layered double hydroxide films with long-term stability on Al substrate, ACS Appl. Mater. Interfaces, 10 (2018) 15150-15162.

[21] D. Jiang, X. Xia, J. Hou, G. Cai, X. Zhang, Z. Dong, A novel coating system with self-reparable slippery surface and active corrosion inhibition for reliable protection of Mg alloy, Chem. Eng. J., 373 (2019) 285-297.

[22] X. Wang, L. Li, Z.-H. Xie, G. Yu, Duplex coating combining layered double hydroxide and 8-quinolinol layers on Mg alloy for corrosion protection, Electrochim. Acta, 283 (2018) 1845-1857.

[23] Z.-H. Xie, L. Wu, Corrosion inhibition of layered double hydroxide coating for Mg alloy in acidic corrosive environments, Mater. Corros., (2019) https://doi.org/10.1002/maco.201910995.

[24] G. Zhang, L. Wu, A. Tang, S. Zhang, B. Yuan, Z. Zheng, F. Pan, A novel approach to fabricate protective layered double hydroxide films on the surface of anodized Mg-Al alloy, Adv Mater Interfaces, 4 (2017) 1700163.

[25] F. Peng, D. Wang, H. Cao, X. Liu, Loading 5-Fluorouracil into calcined Mg/Al layered double hydroxide on AZ31 via memory effect, Mater. Lett., 213 (2018) 383-386.

[26] Y. Zhang, Y. Li, Y. Ren, H. Wang, F. Chen, Double-doped LDH films on aluminum alloys for active protection, Mater. Lett., 192 (2017) 33-35.

[27] H. Li, F. Peng, D. Wang, Y. Qiao, D. Xu, X. Liu, Layered double hydroxide/poly-dopamine composite coating with surface heparinization on Mg alloys: improved anticorrosion, endothelialization and hemocompatibility, Biomater. Sci., 6 (2018) 1846-1858.

[28] Q.-S. Yao, F. Zhang, L. Song, R.-C. Zeng, L.-Y. Cui, S.-Q. Li, Z.-L. Wang, E.-H. Han, Corrosion resistance of a ceria/polymethyltrimethoxysilane modified Mg-Al-layered double hydroxide on AZ31 magnesium alloy, J. Alloys Compd., 764 (2018) 913-928.

[29] H. Qian, D. Xu, C. Du, D. Zhang, X. Li, L. Huang, L. Deng, Y. Tu, J.M.C. Mol, H.A. Terryn, Dual-action smart coatings with a self-healing superhydrophobic surface and anti-corrosion properties, J. Mater. Chem. A, 5 (2017) $2355-2364$.

[30] L. Wu, J. Wu, Z. Zhang, C. Zhang, Y. Zhang, A. Tang, L. Li, G. Zhang, Z. Zheng, A. Atrens, F. Pan, Corrosion resistance of fatty acid and fluoroalkylsilane-modified hydrophobic Mg-Al LDH films on anodized magnesium alloy, Appl. Surf. Sci., 487 (2019) 569-580.

[31] J.A. Yuwono, N. Birbilis, C.D. Taylor, K.S. Williams, A.J. Samin, N.V. Medhekar, Aqueous electrochemistry of the magnesium surface: Thermodynamic and kinetic profiles, Corros. Sci., 147 (2019) 53-68.

[32] D. Seifzadeh, S. Hamzedoust-Hasankiadeh, A.N. Shamkhali, Electrochemical and DFT studies of 8-hydroxyquinoline as corrosion inhibitor for AZ61 magnesium alloy in acidic media, Prot. Met. Phys. Chem. Surf., 49 (2013) 229-239.

[33] M. Zhang, B. Gao, J. Fang, A.E. Creamer, J.L. Ullman, Self-assembly of needle-like layered double hydroxide (LDH) nanocrystals on hydrochar: characterization and phosphate removal ability, RSC Adv., 4 (2014) 28171.

[34] H.W. Ryu, Y.S. Kim, J.H. Kim, I.W. Cheong, Direct synthetic route for water-dispersible polythiophene nanoparticles via surfactant-free oxidative polymerization, Polymer, 55 (2014) 806-812.

[35] E. Ermiş, Synthesis, spectroscopic characterization and DFT calculations of novel Schiff base containing thiophene ring, J. Mol. Struct., 1156 (2018) 91-104.

[36] D. Damazio, E.A. Campos, M.F. Diniz, E.d.C. Mattos, R.d.C.L. Dutra, TLC/IR (UATR) off-line coupling for the characterization of additives in EPDM rubber compositions, Polímeros, 26 (2016) 74-80.

[37] S. Sharifi Golru, M.M. Attar, B. Ramezanzadeh, Studying the influence of nano-Al2O3 particles on the corrosion performance and hydrolytic degradation resistance of an epoxy/polyamide coating on AA-1050, Prog. Org. Coat., 77 (2014) 1391-1399. 
[38] Z. Qiusheng, L. Xiaoyan, Q. Jin, W. Jing, L. Xuegang, Porous zirconium alginate beads adsorbent for fluoride adsorption from aqueous solutions, RSC Adv., 5 (2015) 2100-2112.

[39] B. Deepa, E. Abraham, L.A. Pothan, N. Cordeiro, M. Faria, S. Thomas, Biodegradable Nanocomposite Films Based on Sodium Alginate and Cellulose Nanofibrils, Materials (Basel), 9 (2016).

[40] D. Gu, S. Gao, T. Jiang, B. Wang, Solar-mediated thermo-electrochemical oxidation of sodium dodecyl benzene sulfonate by modulating the effective oxidation potential and pathway for green remediation of wastewater, Sci. Rep., 7 (2017) 44683.

[41] K.R.L. Castagno, V. Dalmoro, D.S. Azambuja, Characterization and corrosion of polypyrrole/sodium dodecylbenzene sulfonate electropolymerised on aluminum alloy 1100, Mater. Chem. Phys., 130 (2011) 721-726.

[42] M. Bouraada, M.S. Ouali, L.C. de Ménorval, Dodecylsulfate and dodecybenzenesulfonate intercalated hydrotalcites as adsorbent materials for the removal of BBR acid dye from aqueous solutions, J. Saudi Chem. Soc., 20 (2016) 397-404.

[43] A.J.C. Silva, S.M.F. Ferreira, D.d.P. Santos, M. Navarro, J. Tonholo, A.S. Ribeiro, A multielectrochromic copolymer based on pyrrole and thiophene derivatives, Sol. Energy Mater. Sol. Cells, 103 (2012) 108-113.

[44] S.H. Patil, A.P. Gaikwad, S.D. Sathaye, K.R. Patil, To form layer by layer composite film in view of its application as supercapacitor electrode by exploiting the techniques of thin films formation just around the corner, Electrochim. Acta, 265 (2018) 556-568.

[45] T. Jiang, W. Malone, Y. Tong, D. Dragoe, A. Bendounan, A. Kara, V.A. Esaulov, Thiophene derivatives on gold and molecular dissociation processes, J. Phys. Chem. C, 121 (2017) 27923-27935.

[46] J.-H. Li, P. Li, J.-T. Xu, C.K. Luscombe, Z.-Q. Fan, Straightening single-walled xarbon nanotubes by adsorbed rigid poly(3-hexylthiophene) chains via $\pi-\pi$ interaction, J. Phys. Chem. C, 120 (2016) 27665-27674.

[47] R. Gan, D. Wang, Z.-H. Xie, L. He, Improving surface characteristic and corrosion inhibition of coating on Mg alloy by trace stannous (II) chloride, Corros. Sci., 123 (2017) 147-157.

[48] M.K. Punith Kumar, M.Y. Rekha, J. Agrawal, T.M. Agarwal, C. Srivastava, Microstructure, morphology and electrochemical properties of ZnFe-Graphene composite coatings, J. Alloys Compd., 783 (2019) 820-827.

[49] R. M Y, C. Srivastava, Microstructure and corrosion properties of zinc-graphene oxide composite coatings, Corros. Sci., 152 (2019) 234-248.

[50] X. Feng, X. Lu, Y. Zuo, D. Chen, The passive behaviour of 304 stainless steels in saturated calcium hydroxide solution under different deformation, Corros. Sci., 82 (2014) 347-355.

[51] X. Feng, X. Lu, Y. Zuo, N. Zhuang, D. Chen, The effect of deformation on metastable pitting of 304 stainless steel in chloride contaminated concrete pore solution, Corros. Sci., 103 (2016) 223-229.

[52] Z.-H. Xie, S. Shan, Nanocontainers-enhanced self-healing Ni coating for corrosion protection of Mg alloy, J. Mater. Sci., 53 (2018) 3744-3755.

[53] H. Zheng, M. Guo, Y. Shao, Y. Wang, B. Liu, G. Meng, Graphene oxide-poly(urea-formaldehyde) composites for corrosion protection of mild steel, Corros. Sci., 139 (2018) 1-12.

[54] M. Mahdavian, M.M. Attar, Another approach in analysis of paint coatings with EIS measurement: Phase angle at high frequencies, Corros. Sci., 48 (2006) 4152-4157.

[55] S. Nezamdoust, D. Seifzadeh, Z. Rajabalizadeh, Application of novel sol-gel composites on magnesium alloy, Journal of Magnesium and Alloys, 7 (2019) 419-432.

[56] M. Heshmati, D. Seifzadeh, P. Shoghi, M. Gholizadeh-Gheshlaghi, Duplex Ni-Zn-Cu-P/Ni-P electroless coating on magnesium alloy via maleic acid pretreatment, Surf. Coat. Technol., 328 (2017) 20-29.

[57] J.K. Lin, C.L. Hsia, J.Y. Uan, Characterization of Mg,Al-hydrotalcite conversion film on $\mathrm{Mg}$ alloy and $\mathrm{Cl}^{-}$and $\mathrm{CO}_{3}{ }^{2-}$ anion-exchangeability of the film in a corrosive environment, Scripta Mater., 56 (2007) 927-930. 
Tables:

Table 1 Peak assignments on the FT-IR spectra of MBT@MgAl-LDH, SA@MgAl-LDH, SDBS@MgAl-LDH, and NTA@MgAl-LDH coatings.

\begin{tabular}{|c|c|c|}
\hline Samples & Wave number $/ \mathrm{cm}^{-1}$ & Assignments \\
\hline b2: MBT@MgAl-LDH[36, & 3062 & phenyl ring $\mathrm{C}-\mathrm{H}$ stretching vibrations \\
\hline \multirow[t]{3}{*}{ 37] } & 1453 & phenyl ring $\mathrm{C}=\mathrm{C}$ stretching vibrations \\
\hline & 1008 & the $\mathrm{C}=\mathrm{N}$ band intensity \\
\hline & 749 & out-of-plane bending vibration of $\mathrm{ArC}-\mathrm{H}$ bond \\
\hline \multirow[t]{2}{*}{ b3:SA@MgAl-LDH[38, 39] } & 1444 & $\mathrm{COO}-$ stretching vibration \\
\hline & 1034 & $\mathrm{C}-\mathrm{O}$ stretching vibration of polysaccharide \\
\hline b4: & 1449 & the $\mathrm{C}=\mathrm{C}$ stretching vibrations of the benzene \\
\hline SDBS@MgAl-LDH[40-42] & 1188 & $\mathrm{~S}=\mathrm{O}$ stretching vibration \\
\hline b5: NTA@MgAl-LDH[34, & 3084 & $\mathrm{C}-\mathrm{H}$ stretching vibration of the thiophene ring \\
\hline \multirow[t]{5}{*}{$35,43]$} & 2919 & $\mathrm{C}-\mathrm{H}$ stretching vibration of the alkyl group \\
\hline & 2852 & \\
\hline & 1472 & $\mathrm{C}=\mathrm{C}$ stretching vibration of the thiophene ring \\
\hline & 830 & C-S-C stretching vibration of the thiophene ring \\
\hline & 758 & \\
\hline
\end{tabular}




\section{Journal Pre-proof}

Table 2 Fitting electrochemical parameters for EIS spectra of different MgAl-LDH coatings

\begin{tabular}{|c|c|c|c|c|c|c|c|c|c|c|}
\hline Coatings & $\begin{array}{c}R_{\mathrm{s}} / \\
\Omega \mathrm{cm}^{2}\end{array}$ & $\begin{array}{c}Y_{2} / 10^{-12} \\
\mathrm{~S} \mathrm{~s}^{\mathrm{n}} \mathrm{cm}^{-2}\end{array}$ & $\begin{array}{l}R_{2} / \\
\mathrm{M} \Omega \\
\mathrm{cm}^{2} \\
\end{array}$ & $\begin{array}{c}Y_{1} / 10^{-11} \\
\mathrm{~S} \mathrm{~s}^{\mathrm{n}} \mathrm{cm}^{-2}\end{array}$ & $\begin{array}{c}R_{1} / \\
\mathrm{M} \Omega \mathrm{cm}^{2}\end{array}$ & $\begin{array}{c}Y_{\mathrm{dl}} / 10^{-8} \\
\mathrm{~S} \mathrm{~s}^{\mathrm{n}} \mathrm{cm}^{-2}\end{array}$ & $\begin{array}{l}R_{\mathrm{ct}} / \\
\mathrm{M} \Omega \\
\mathrm{cm}^{2} \\
\end{array}$ & $\begin{array}{c}Z_{\mathrm{W}} / 10^{-7} \\
\mathrm{~S} \mathrm{~s}^{0.5} \\
\mathrm{~cm}^{-2}\end{array}$ & $\begin{array}{c}|\mathrm{Z}|_{f=0.1 \mathrm{~Hz}} / \\
\mathrm{M} \Omega \mathrm{cm}^{2}\end{array}$ & $\begin{array}{c}\chi^{2 /} \\
10^{-4}\end{array}$ \\
\hline MgAl-LDH & 4 & N/A & N/A & 2.862 & 1.030 & 2.808 & 22.16 & 2.815 & 15.63 & 9.07 \\
\hline $\begin{array}{c}\text { MBT@ } \\
\text { MgAl-LDH }\end{array}$ & 5 & 7.735 & 1.109 & 0.5804 & 13.55 & 1.259 & 102.6 & N/A & 67.94 & 3.59 \\
\hline $\begin{array}{c}\text { SA@ } \\
\text { MgAl-LDH }\end{array}$ & 4 & 1.112 & 4.939 & 0.6049 & 142.6 & 0.03766 & 713.1 & N/A & 592.9 & 2.15 \\
\hline $\begin{array}{c}\text { SDBS@ } \\
\text { MgAl-LDH }\end{array}$ & 4.5 & 8.519 & 7.573 & 5.315 & 29.14 & 0.3077 & 883.5 & N/A & 315.9 & 3.76 \\
\hline $\begin{array}{c}\text { NTA@ } \\
\text { MgAl-LDH }\end{array}$ & 6.5 & 5.531 & 7.910 & 6.925 & 614.2 & 0.1575 & 1837 & N/A & 1097.7 & 6.62 \\
\hline
\end{tabular}




\section{Journal Pre-proof}

Table 3 Electrochemical parameters based on the potentiodynamic polarization curves

\begin{tabular}{ccccc}
\hline Samples & $\beta_{\mathrm{a}} / \mathrm{mV} \mathrm{dec}^{-1}$ & $-\beta_{\mathrm{c}} / \mathrm{mV} \mathrm{dec}^{-1}$ & $E_{\text {corr }} / \mathrm{V}$ & $j_{\text {corr }} / \mathrm{nA} \mathrm{cm}^{-2}$ \\
\hline Mgalloy & 369 & 94 & -1.50 & 4327 \\
MgAl-LDH & 205 & 196 & -0.750 & 1.543 \\
MBT@MgAl-LDH & 198 & 199 & -0.623 & 0.3976 \\
SA@MgAl-LDH & 207 & 206 & -0.658 & 0.06215 \\
SDBS@MgAl-LDH & 187 & 210 & -0.674 & 0.06141 \\
NTA@MgAl-LDH & 194 & 190 & -0.437 & 0.04001 \\
\hline
\end{tabular}


Figures and Figure Captions:
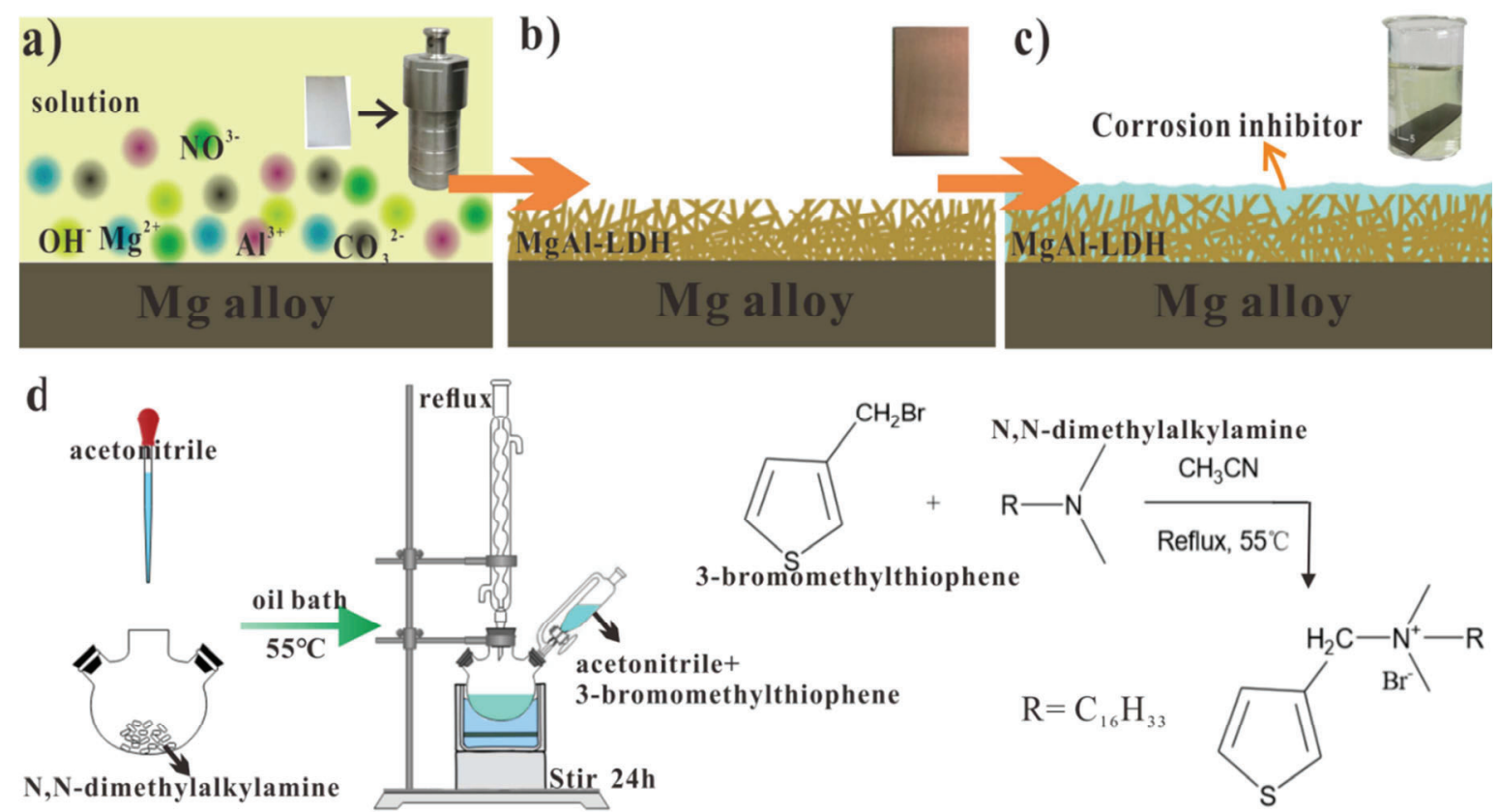

Scheme 1. The schematic diagrams of the (a, b, and c) in-situ growth of MgAl-LDH film and the preparation of CIs@MgAl-LDH coatings on Mg alloy, and (d) synthesis of NTA inhibitor. 

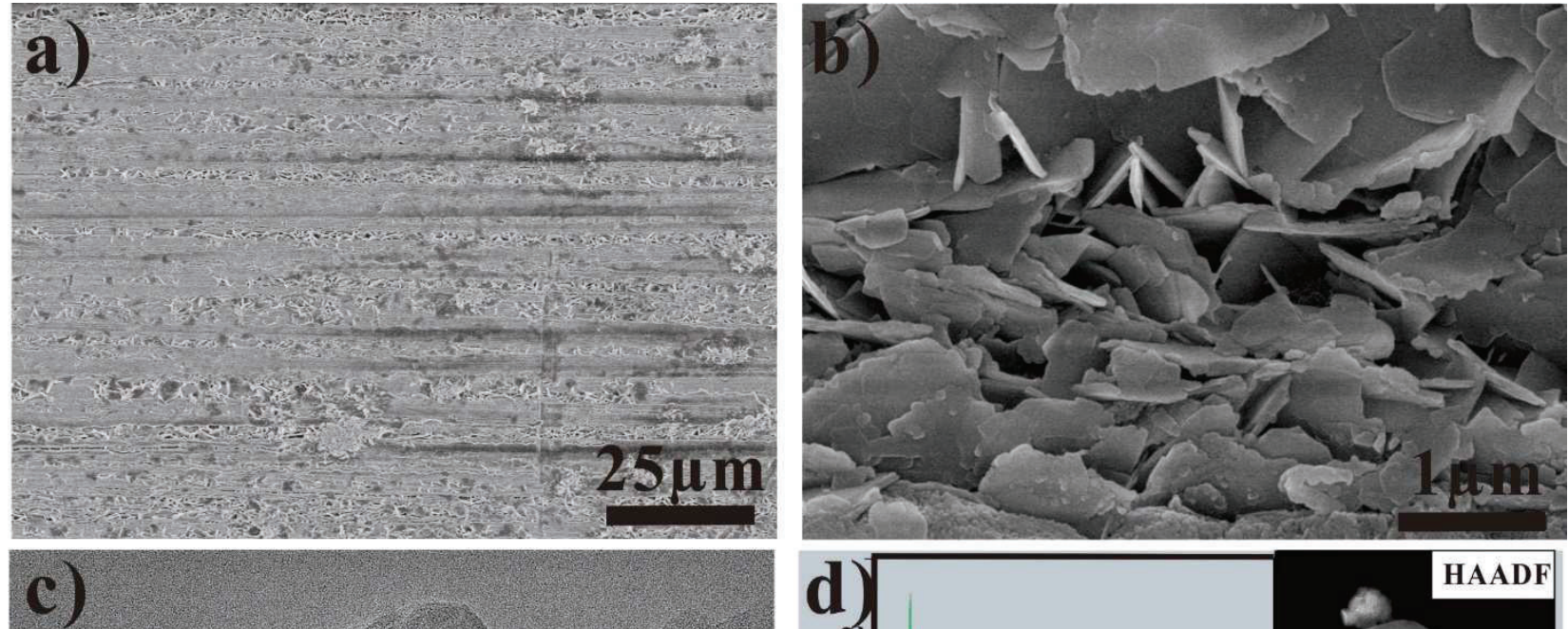

c)
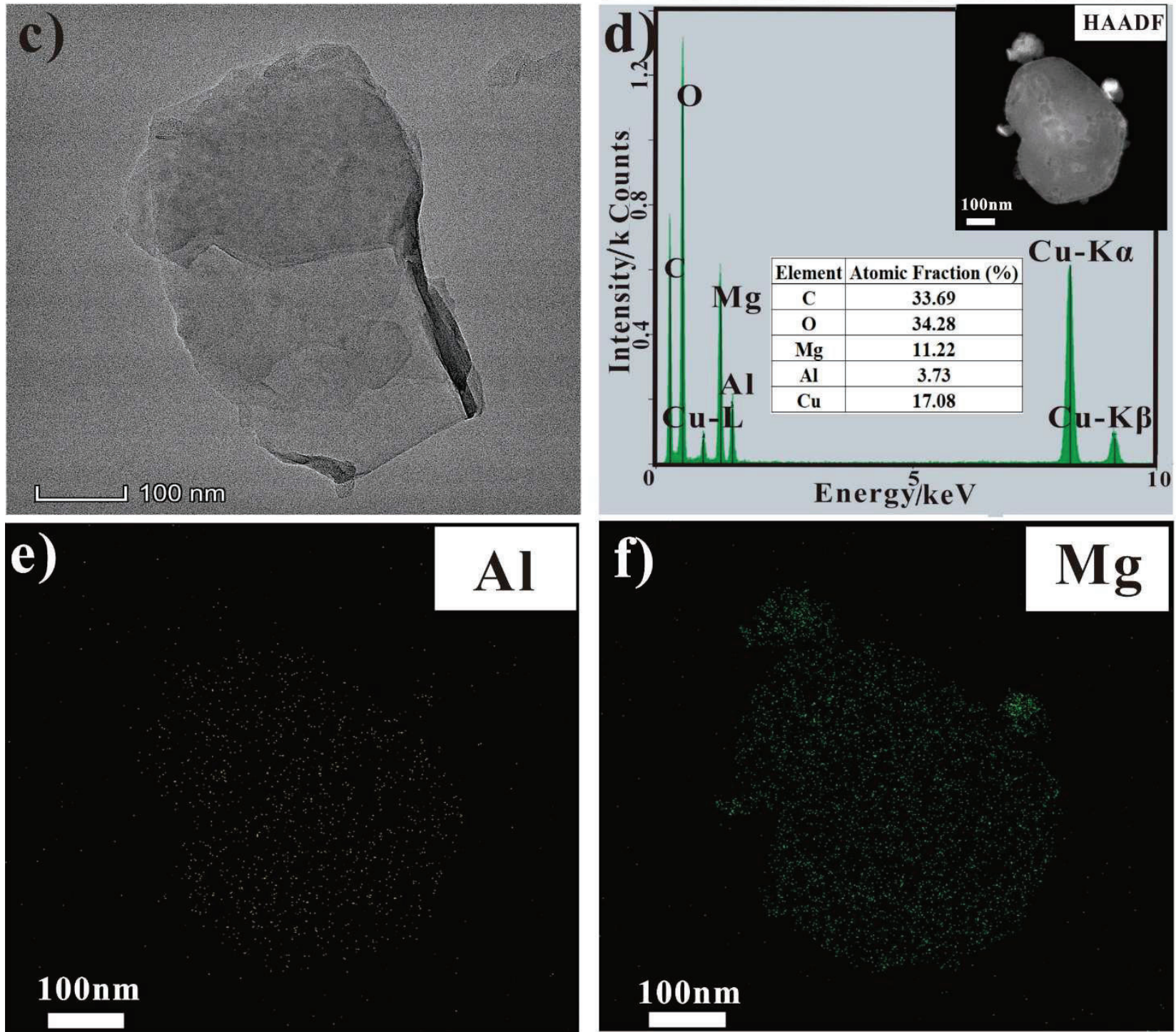

Figure 1. (a, b) SEM and (c) TEM of MgAl-LDH depositions, and (d, e, f) the corresponding HAADF-STEM-EDS spectra and maps showing the full and uniform distribution of $\mathrm{Al}$ and $\mathrm{Mg}$. 

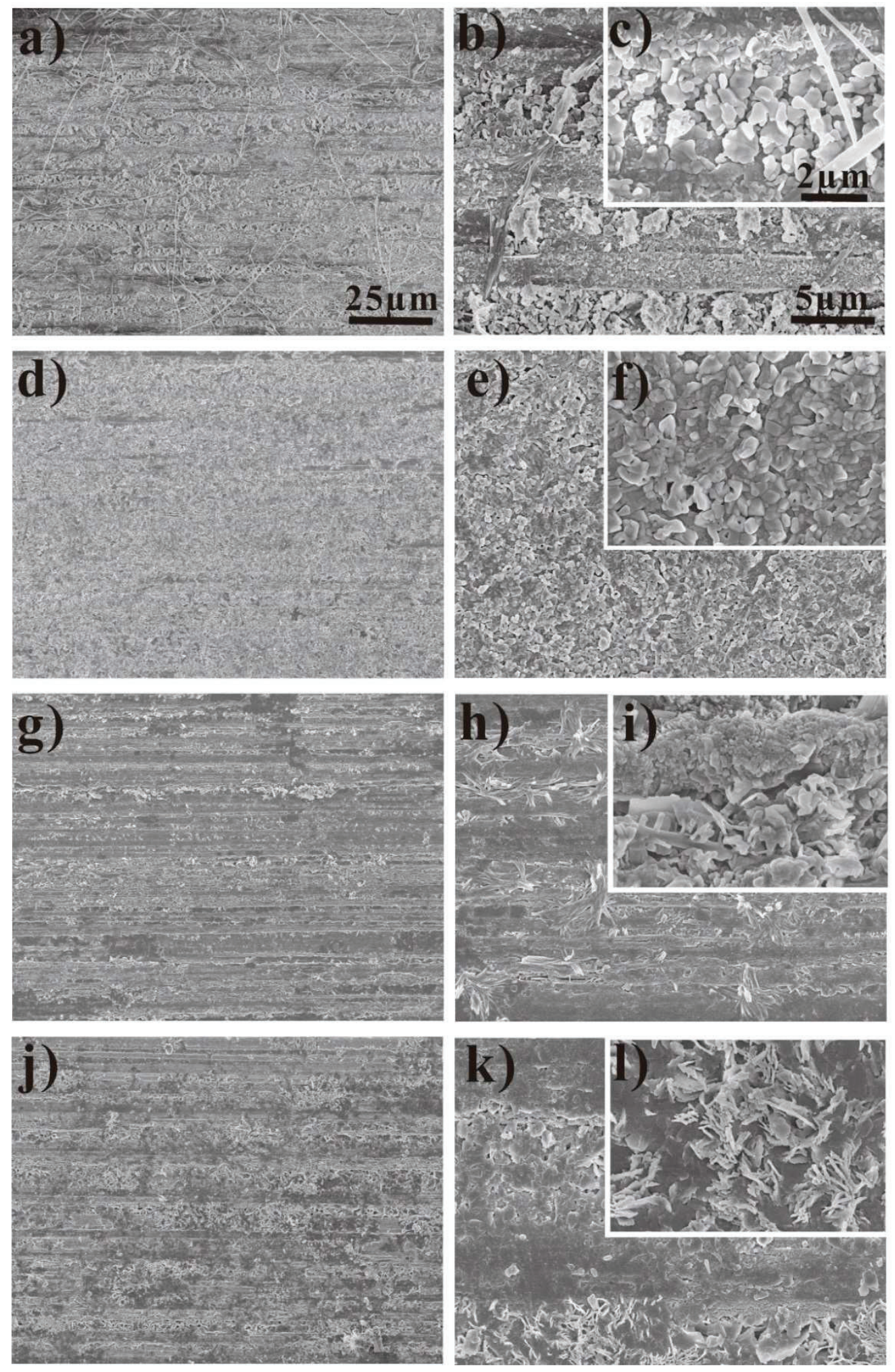

Figure 2. SEM images illustrating the surface morphologies of the MgAl-LDH layer after modification with different CIs at different magnifications. (a, b, and c) MBT@MgAl-LDH, (d, e, and f) SA@MgAl-LDH, (g, h, and i) SDBS@MgAl-LDH, and (j, k, and l) NTA@MgAl-LDH coatings. The graphs in the left and right columns and the insets have identical scale bars, respectively. 


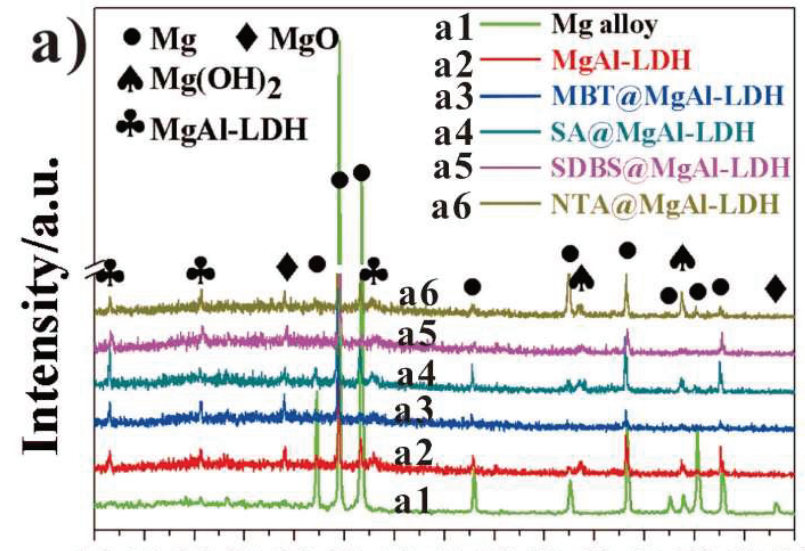

101520253035404550556065707580

2 Theta/degree

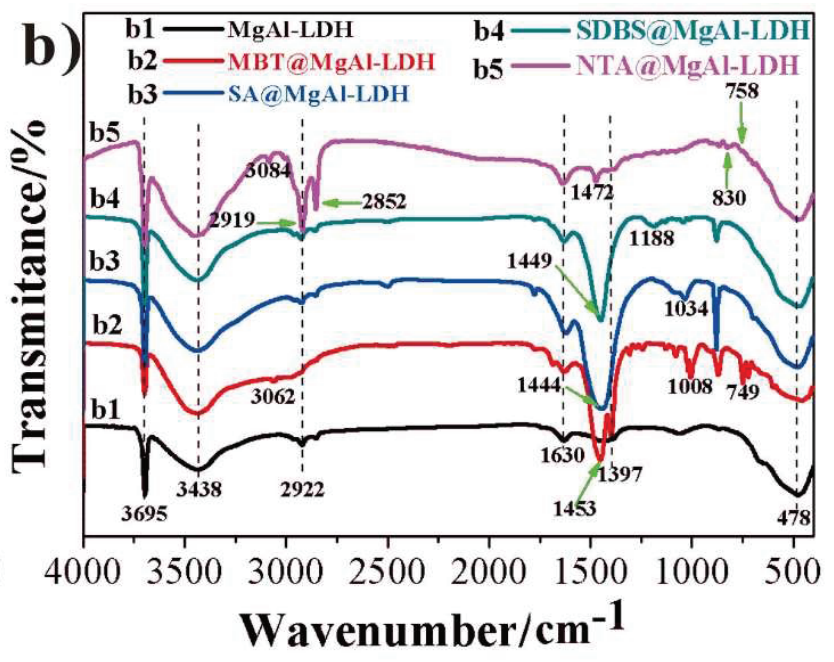

Figure 3. (a) XRD patterns and (b) FT-IR spectra of the (a1) Mg alloy, (a2, b1) MgAl-LDH, (a3, b2) MBT@MgAl-LDH, (a4, b3) SA@MgAl-LDH, (a5, b4) SDBS@MgAl-LDH, and (a6, b5) NTA@MgAl-LDH coatings. 


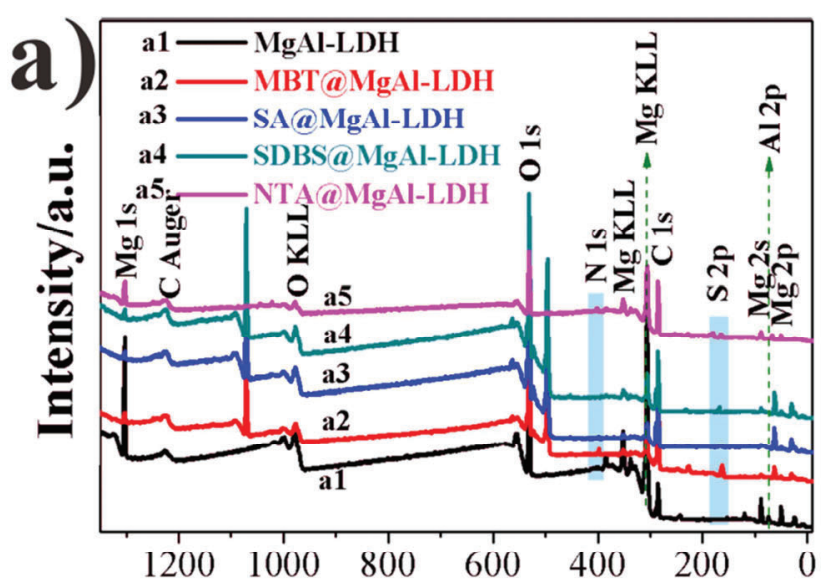

Binding Energy/eV

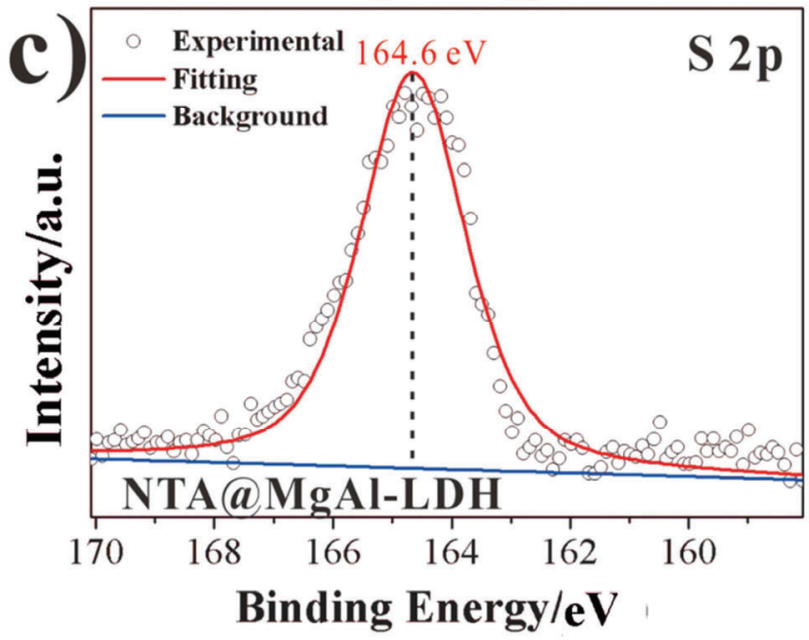

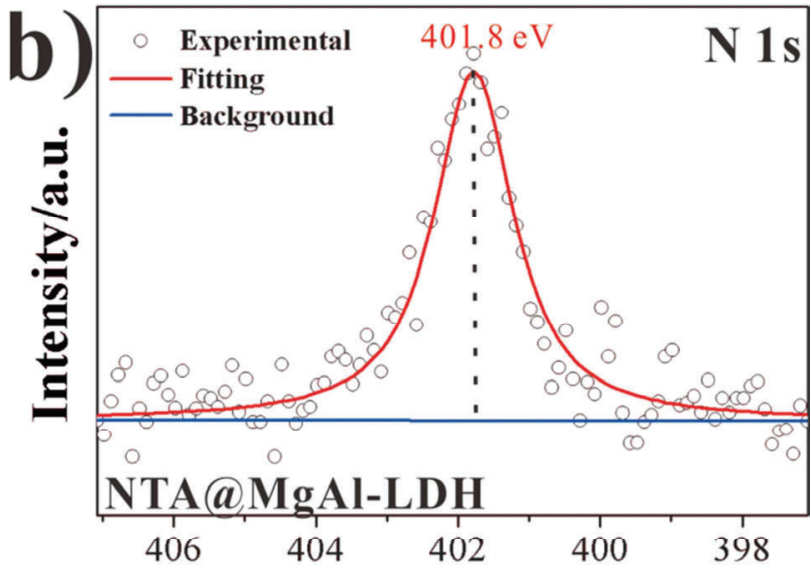

Binding Energy/eV

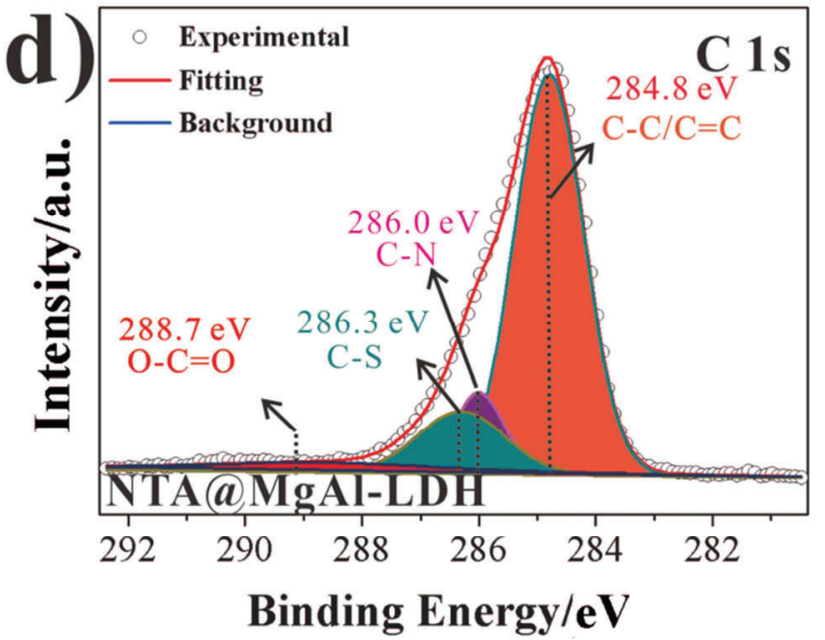

Figure 4. (a) XPS survey spectrum of the (a1) MgAl-LDH, (a2) MBT@MgAl-LDH, (a3) SA@MgAl-LDH, (a4) SDBS@MgAl-LDH and (a5) NTA@MgAl-LDH coating. The high resolution XPS spectra of (b) N 1s, (c) S 2p, and (d) C 1s for NTA@MgAl-LDH coating. 

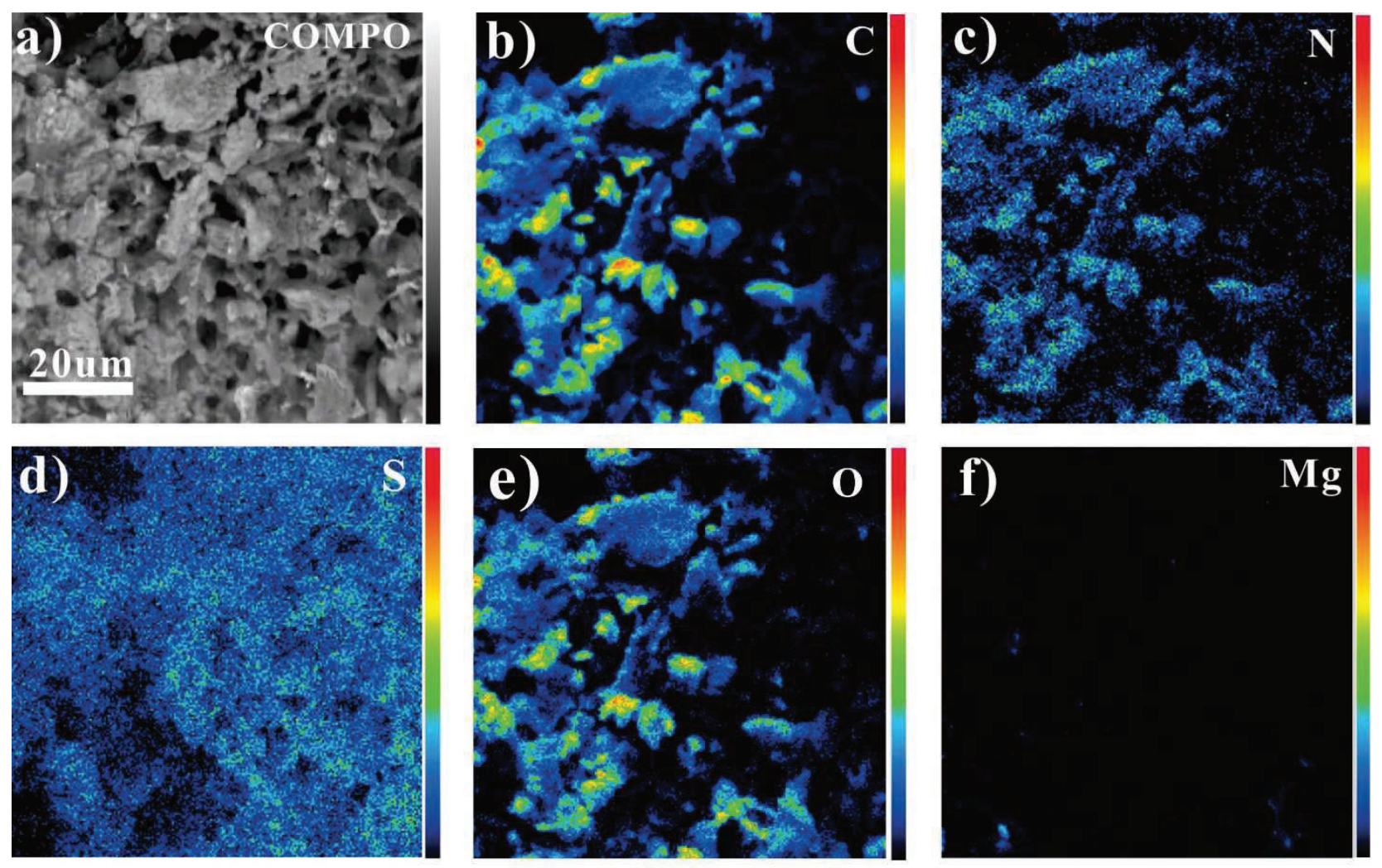

Figure 5. Results of EPMA-WDS mapping analysis showing the elemental distribution of $\mathrm{C}, \mathrm{N}, \mathrm{S}, \mathrm{O}$, and $\mathrm{Mg}$ on the surface of the NTA@MgAl-LDH coating. 

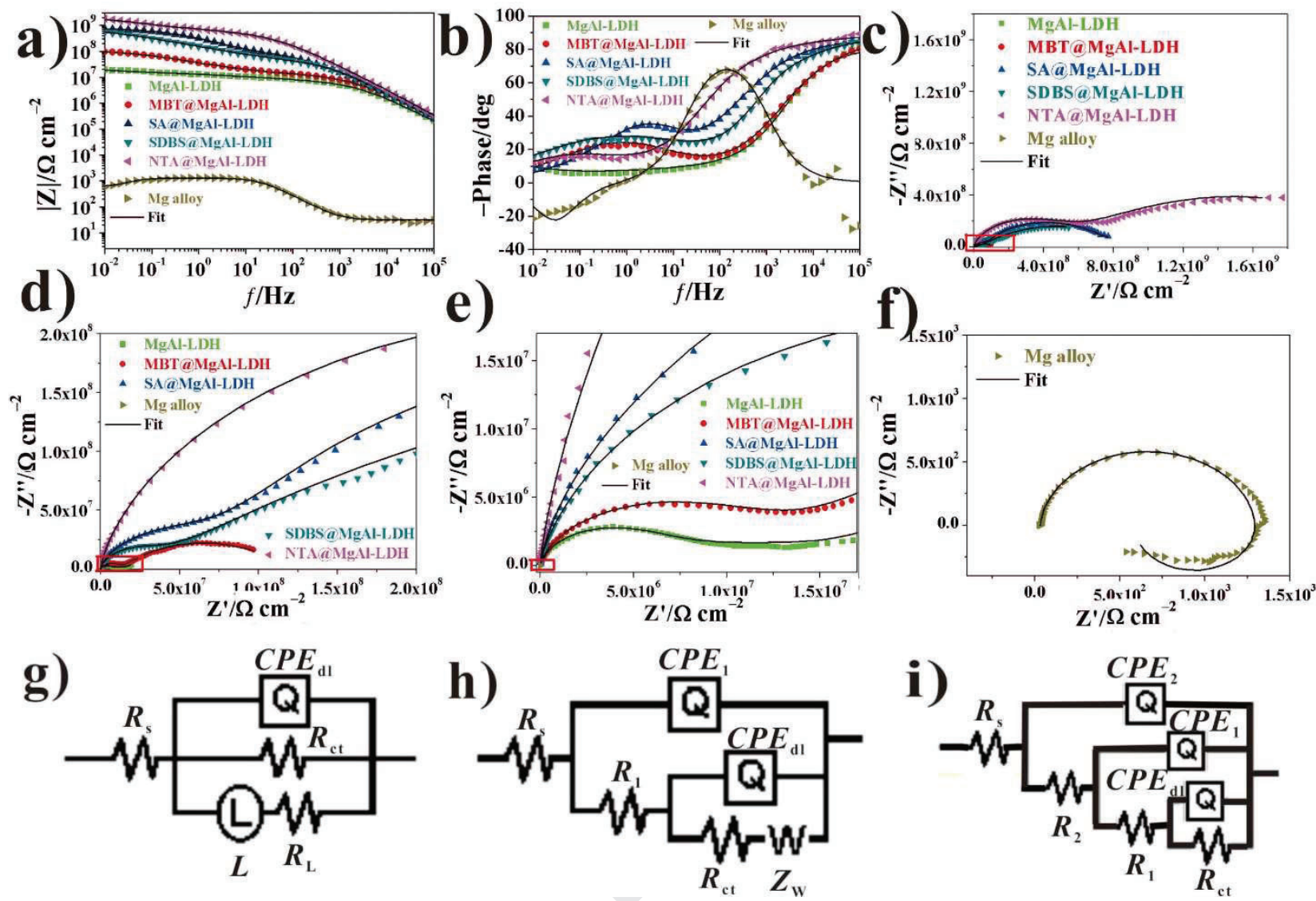

Figure 6. EIS diagrams of Mg alloy, MgAl-LDH, and CIs@MgAl-LDH coatings in 3.5 wt.\% NaCl solution. (a, b) Bode magnitude and Bode phase plots. (c) Nyquist plots. (d) Magnified Nyquist plots for the square area in (c). (e) Magnified Nyquist plots for the square area in (d). (f) Nyquist plots for AZ31 Mg alloy substrate. The dotted and solid lines are experimental and fitting results, respectively. Equivalent circuits of (g) AZ31 Mg alloy substrate, (h) MgAl-LDH coating, and (i) CIs@MgAl-LDH coatings. 


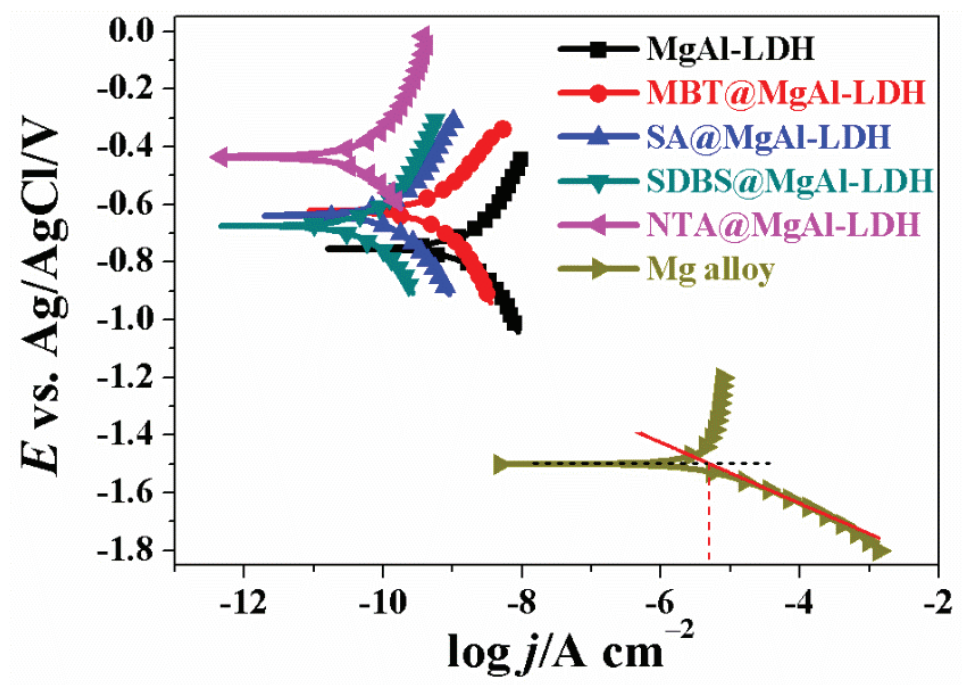

Figure 7. Potentiodynamic polarization curves of $\mathrm{Mg}$ alloy, $\mathrm{MgAl}-\mathrm{LDH}$ and different $\mathrm{CIs} @ \mathrm{MgAl}-\mathrm{LDH}$ coatings in 3.5 wt. $\% \mathrm{NaCl}$ medium. 


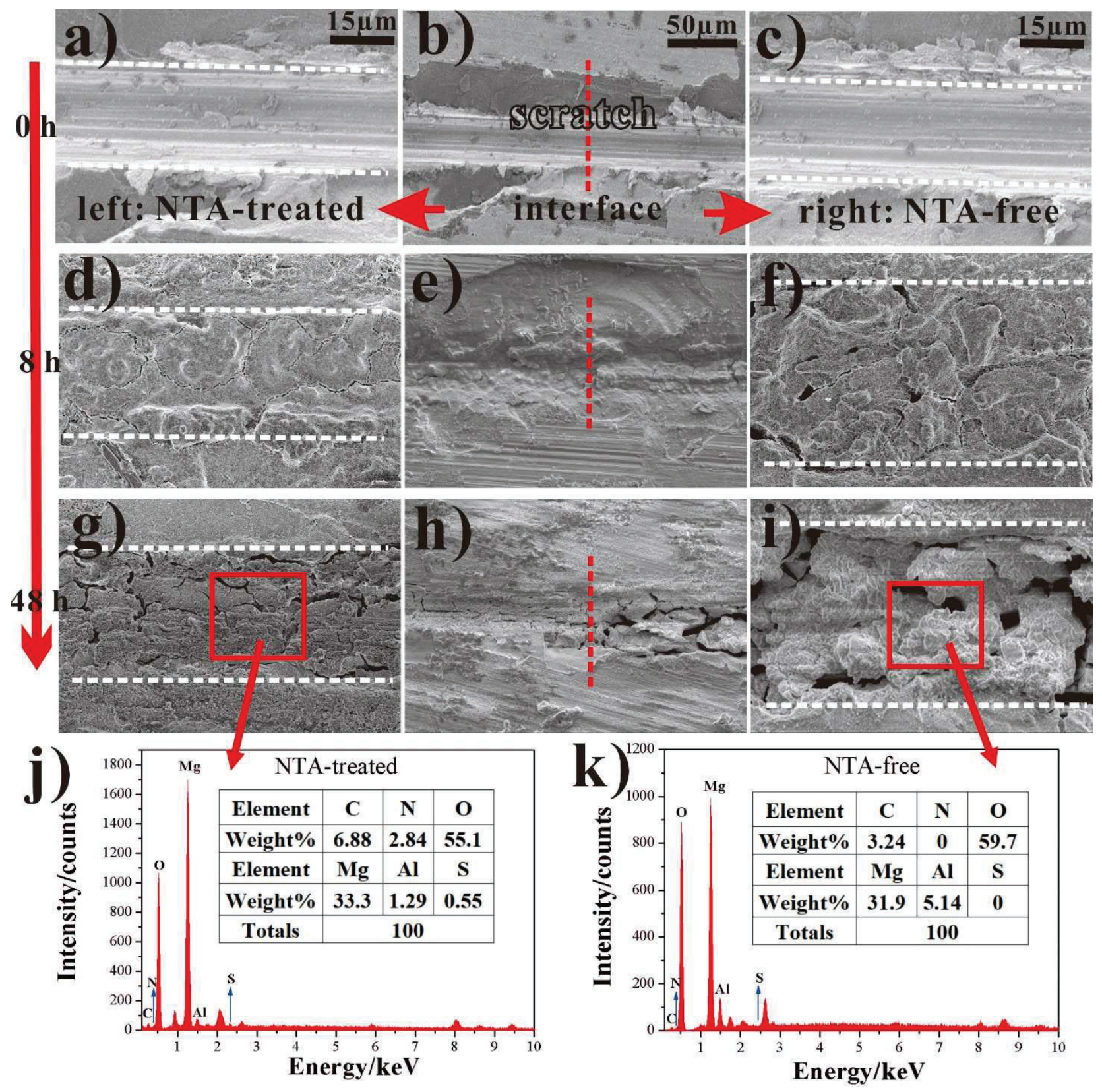

Figure 8. SEM images showing the morphological evolutions of MgAl-LDH coating with and without modification by NTA after artificial scratch and soaking in 3.5 wt.\% $\mathrm{NaCl}$ solution. (a, b, c) Fresh sample after scratch, (d, e, f) 8 h, and $(\mathrm{g}, \mathrm{h}, \mathrm{f}) 48 \mathrm{~h}$ of immersion. The graphs in the left column shows the area treated with NTA (a, d, g), the middle column is the interface including both NTA-treated and NTA-free areas (b, e, h); those in the right column is the area without NTA treatment (c, f, i). Middle column: 500 times; left and right column: 1500 times. EDS of NTA-treated (j) and NTA-free $(\mathrm{k})$ areas of MgAl-LDH coating after $48 \mathrm{~h}$ of immersion. 


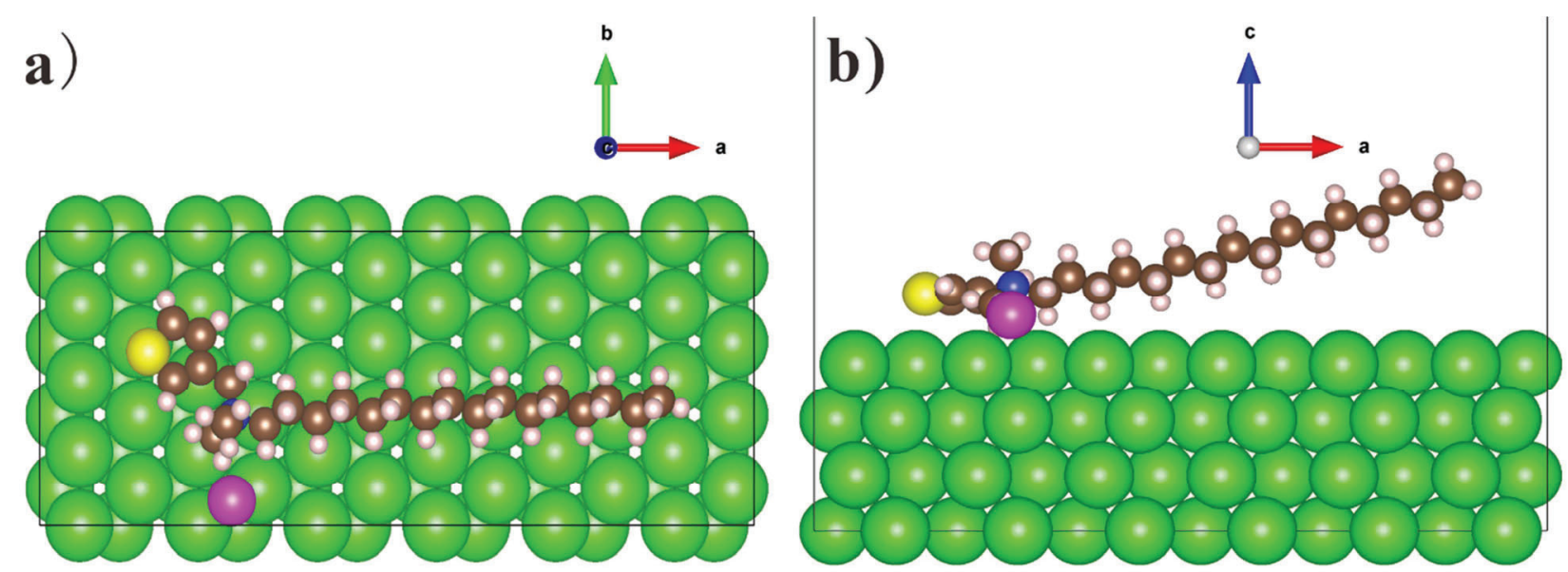

Figure 9. (a) Top and (b) side views of computational models for the optimized adsorption structure of NTA molecule onto the Mg (0001) surface with four layers. The green, brown, white, blue, yellow and magenta balls stand for the $\mathrm{Mg}, \mathrm{C}, \mathrm{H}, \mathrm{N}, \mathrm{S}$ and $\mathrm{Br}$ atoms, respectively. 


\section{Highlights}

$>$ Intelligent anticorrosion coatings consist of $\mathrm{LDH}$ and inhibitor layers were obtained.

$>$ As-synthesized NTA inhibitor can effective boost corrosion protection of LDH film.

$>$ Anticorrosion properties of several inhibitor-treated LDH coatings are given.

$>$ Adsorption structure of NTA molecule on the $\mathrm{Mg}$ (0001) surface was optimized. 


\section{Declaration of interests}

$\bigotimes$ The authors declare that they have no known competing financial interests or personal relationships that could have appeared to influence the work reported in this paper.

$\square$ The authors declare the following financial interests/personal relationships which may be considered as potential competing interests: 


\section{Supporting Information}

The Development of a Thiophene Derivative Modified LDH Coating for Mg Alloy Corrosion Protection

Lin-Xin $\mathrm{Li}^{\mathrm{a}}$, Zhi-Hui Xie ${ }^{\mathrm{a}, \mathrm{e},}$, Carlos Fernandez ${ }^{\mathrm{b}}$, Liang $\mathrm{Wu}^{\mathrm{c}}$, Daojian Cheng ${ }^{\mathrm{d}}$,

Xiao-Hui Jiang ${ }^{\text {a }}$, Chuan-Jian Zhong ${ }^{e}$

${ }^{\text {a}}$ Chemical Synthesis and Pollution Control Key Laboratory of Sichuan Province/Institute of Synthesis and Application of Functional Materials, College of Chemistry and Chemical Engineering, China West Normal University, Nanchong, 637002, P.R. China.

${ }^{b}$ School of Pharmacy and Life Sciences, Robert Gordon University, Garthdee Road, Aberdeen, AB107GJ United Kingdom

${ }^{\mathrm{c}}$ College of Materials Science and Engineering/National Engineering Research Center for Magnesium Alloys, Chongqing University, Chongqing 400044, P.R. China

${ }^{\mathrm{d}}$ Key Laboratory of Organic-Inorganic Composites, Beijing University of Chemical Technology, Beijing 100029, P.R. China

${ }^{\circ}$ Department of Chemistry, State University of New York at Binghamton, Binghamton, New York 13902, United States

${ }^{*}$ Corresponding authors:

Emails: zhxie@.cwnu.edu.cn (Z.-H. Xie) 


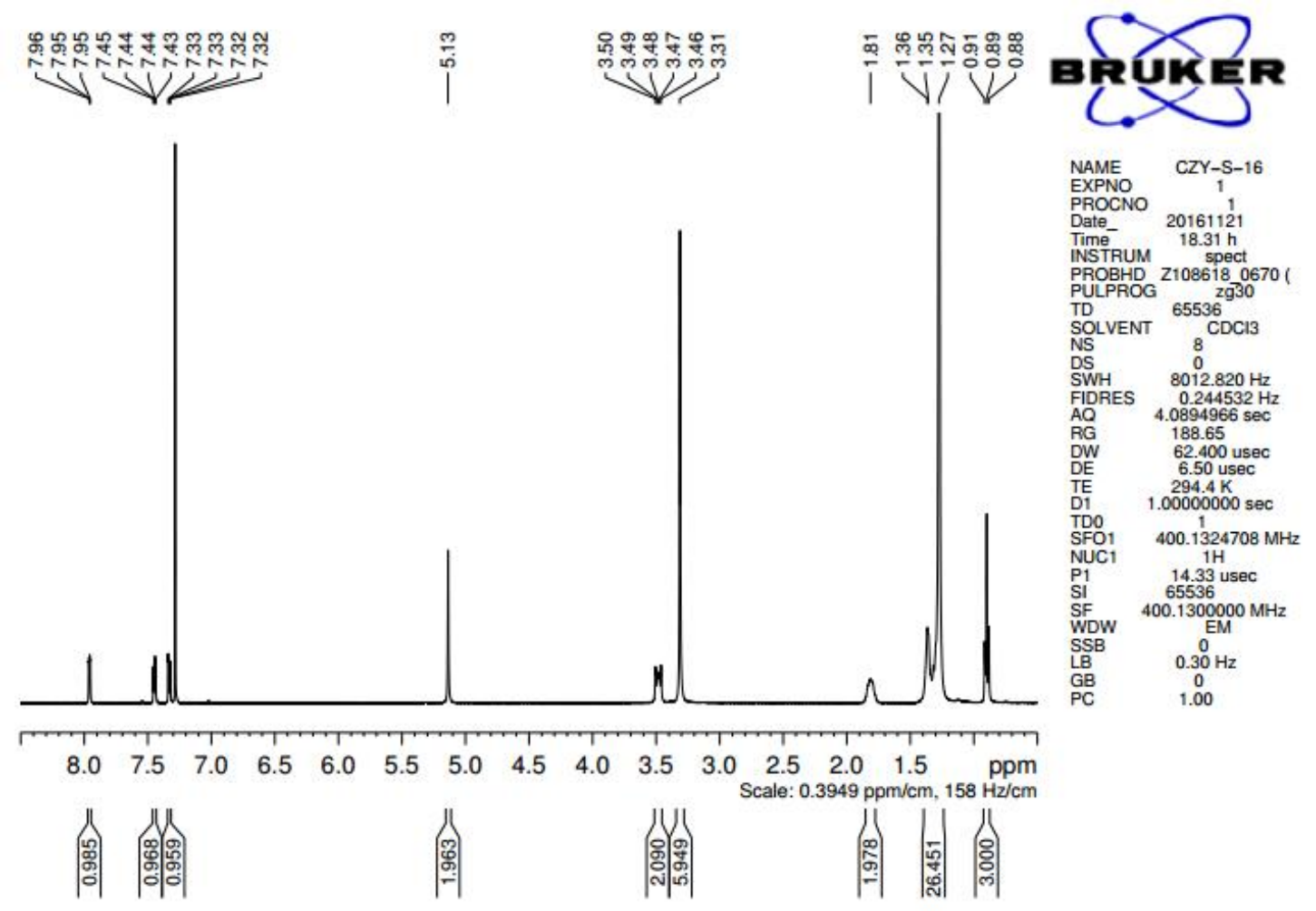

Figure S1 ${ }^{1} \mathrm{H}$ NMR for N-alkyl-N, N-dimethyl-N-(3-thienylmethylene) ammonium bromides (NTA) 
Table S1 The ${ }^{1} \mathrm{H}$ NMR analysis of NTA

\begin{tabular}{cccl}
\hline$\delta(\mathrm{ppm})$ & multiplicity & number & \multicolumn{1}{c}{ type of $\mathrm{C}-\mathrm{H}$} \\
\hline $7.96-7.95$ & $\mathrm{~m}$ & $1 \mathrm{H}$ & $\mathrm{H}$ of thiophene ring \\
$7.45-7.43$ & $\mathrm{~m}$ & $1 \mathrm{H}$ & \\
$7.33-7.32$ & $\mathrm{~m}$ & $1 \mathrm{H}$ & \\
5.13 & $\mathrm{~s}$ & $2 \mathrm{H}$ & $\mathrm{H}$ of $\mathrm{CH}_{2}$ attached to the thiophene ring \\
$3.50-3.46$ & $\mathrm{t}$ & $2 \mathrm{H}$ & $\mathrm{H}$ of $\alpha-\mathrm{CH}_{2}$ of hexadecyl linked to nitrogen \\
3.31 & $\mathrm{~s}$ & $6 \mathrm{H}$ & $\mathrm{H}$ of two $\mathrm{CH}_{3}$ on nitrogen \\
1.81 & $\mathrm{~m}$ & $2 \mathrm{H}$ & $\mathrm{H}$ of $\beta-\mathrm{CH}_{2}$ of cetyl linked to nitrogen \\
$1.36-1.27$ & $\mathrm{~m}$ & $26 \mathrm{H}$ & $\mathrm{H}$ other than the ends of hexadecyl \\
$0.91-0.88$ & $\mathrm{t}$ & $3 \mathrm{H}$ & $\mathrm{H}$ of $\mathrm{CH}_{3}$ at the hexadecyl end \\
\hline
\end{tabular}




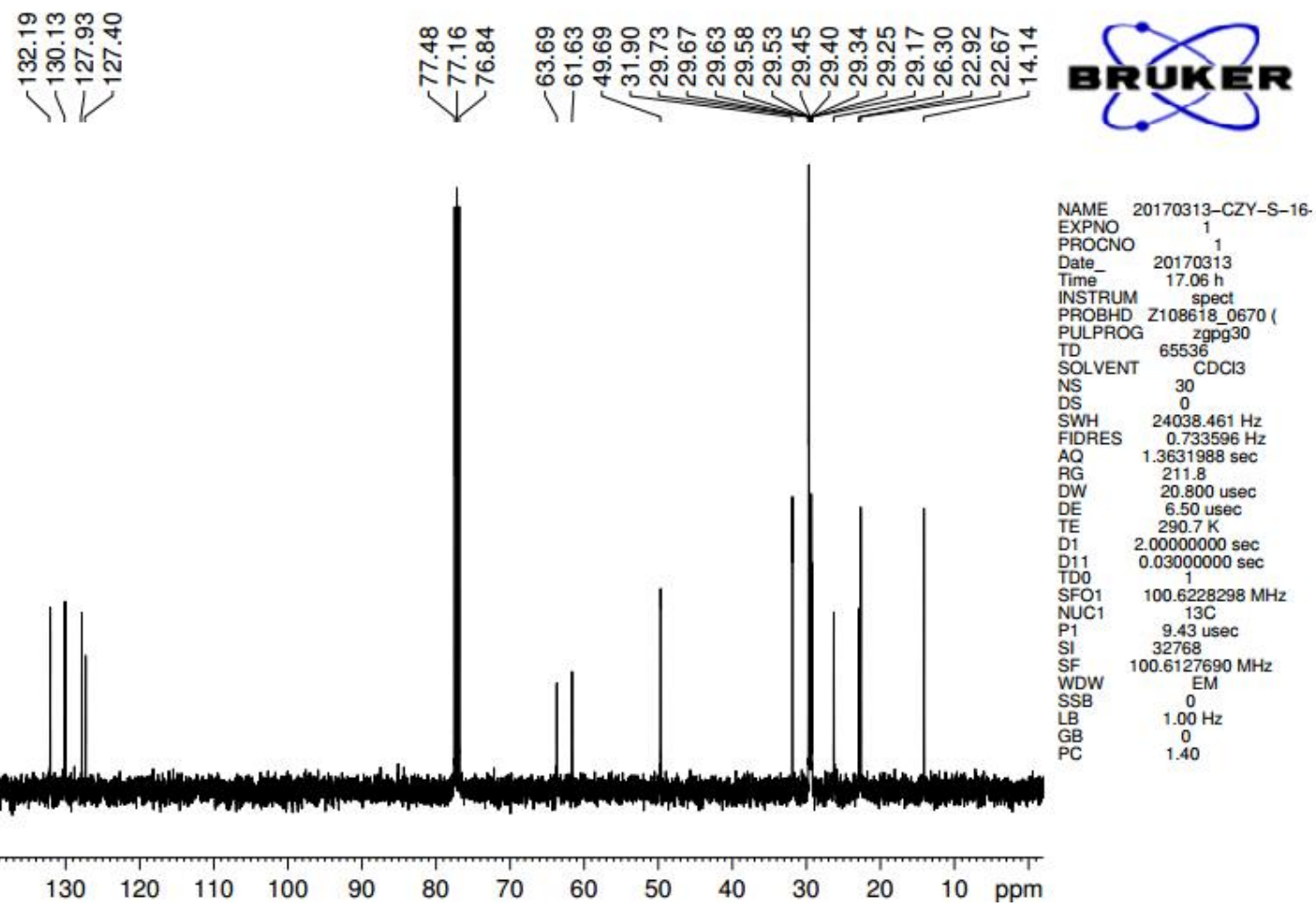

Figure S2 ${ }^{13} \mathrm{C}$ NMR for NTA 
Table S2 The ${ }^{13} \mathrm{C}$ NMR analysis of NTA

\begin{tabular}{cl}
\hline$\delta(\mathrm{ppm})$ & \multicolumn{1}{c}{ type of $\mathrm{C}$} \\
\hline $132.19,130.13,127.93,127.40$ & $\mathrm{C}$ on the thiophene ring \\
63.69 & $\alpha-\mathrm{C}$ of hexadecyl linked to nitrogen \\
61.63 & $\mathrm{C}$ of $\mathrm{CH}_{2}$ attached to the thiophene ring \\
49.69 & $\mathrm{C}$ of two $\mathrm{CH}_{3}$ linked on nitrogen \\
$31.90,29.73,29.67,29.63,29.58,29.53,29.45$, & the $\mathrm{C}$ peak of the remaining fifteen carbons of \\
$29.40,29.34,29.25,29.17,26.30,22.92,22.67$, & hexadecyl \\
14.14 & \\
\hline
\end{tabular}




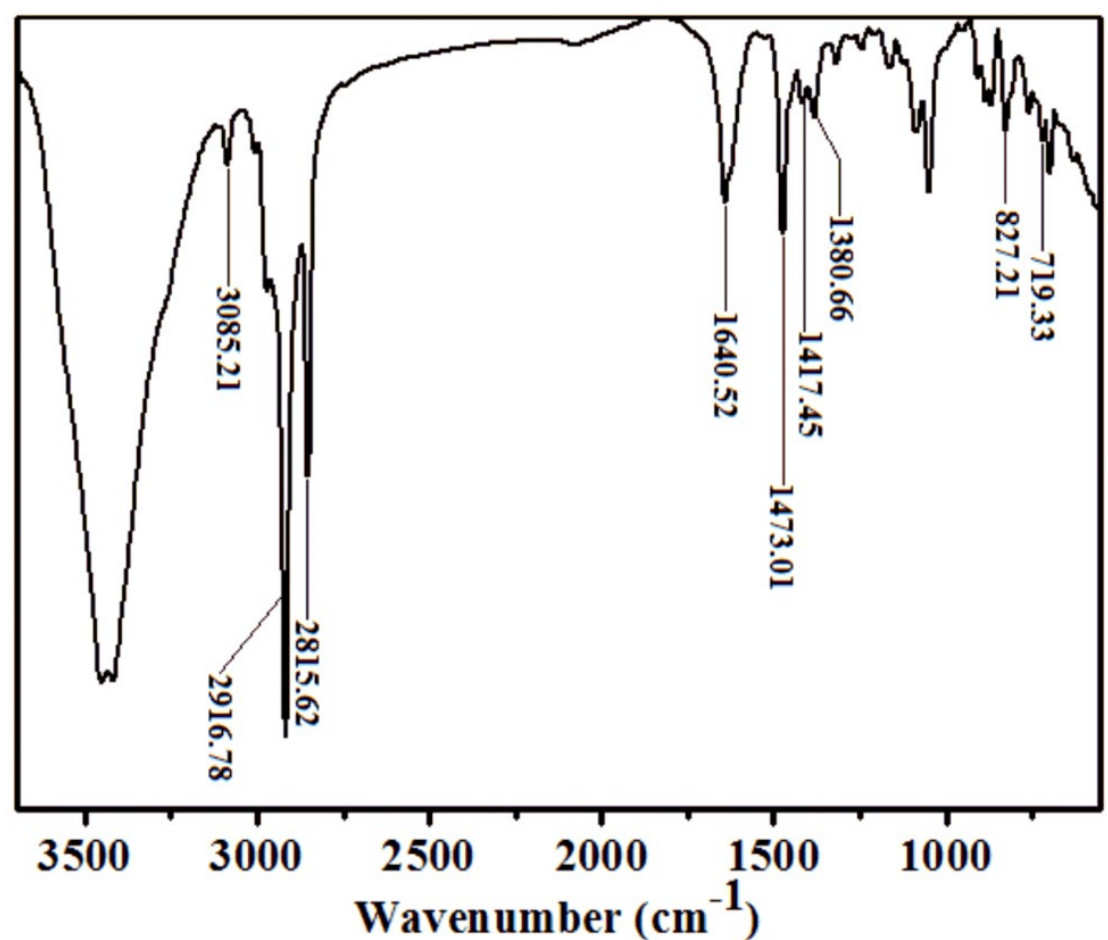

Figure S3 FT-IR spectra for NTA 
Table S3 The FT-IR spectra analysis of NTA

\begin{tabular}{cl}
\hline Wave number $\left(\mathrm{cm}^{-1}\right)$ & \multicolumn{1}{c}{ Assignments } \\
\hline 3085.21 & $\mathrm{C}-\mathrm{H}$ stretching vibration of thiophene ring \\
$2916.78,2815.62$, & $\mathrm{C}-\mathrm{H}$ stretching vibration of alkyl group \\
$1640.62,1473.01$ & $\mathrm{C}=\mathrm{C}$ stretching vibration of thiophene ring \\
1417.45 & $\mathrm{C}-\mathrm{H}$ in-plane bending vibration of thiophene ring \\
1380.66 & $\mathrm{CH}$ bending vibration \\
827.61 & $\mathrm{C}-\mathrm{S}-\mathrm{C}$ stretching vibration of thiophene ring \\
719.33 & \\
\hline
\end{tabular}




\section{Mass Spectrum SmartFormula Report}

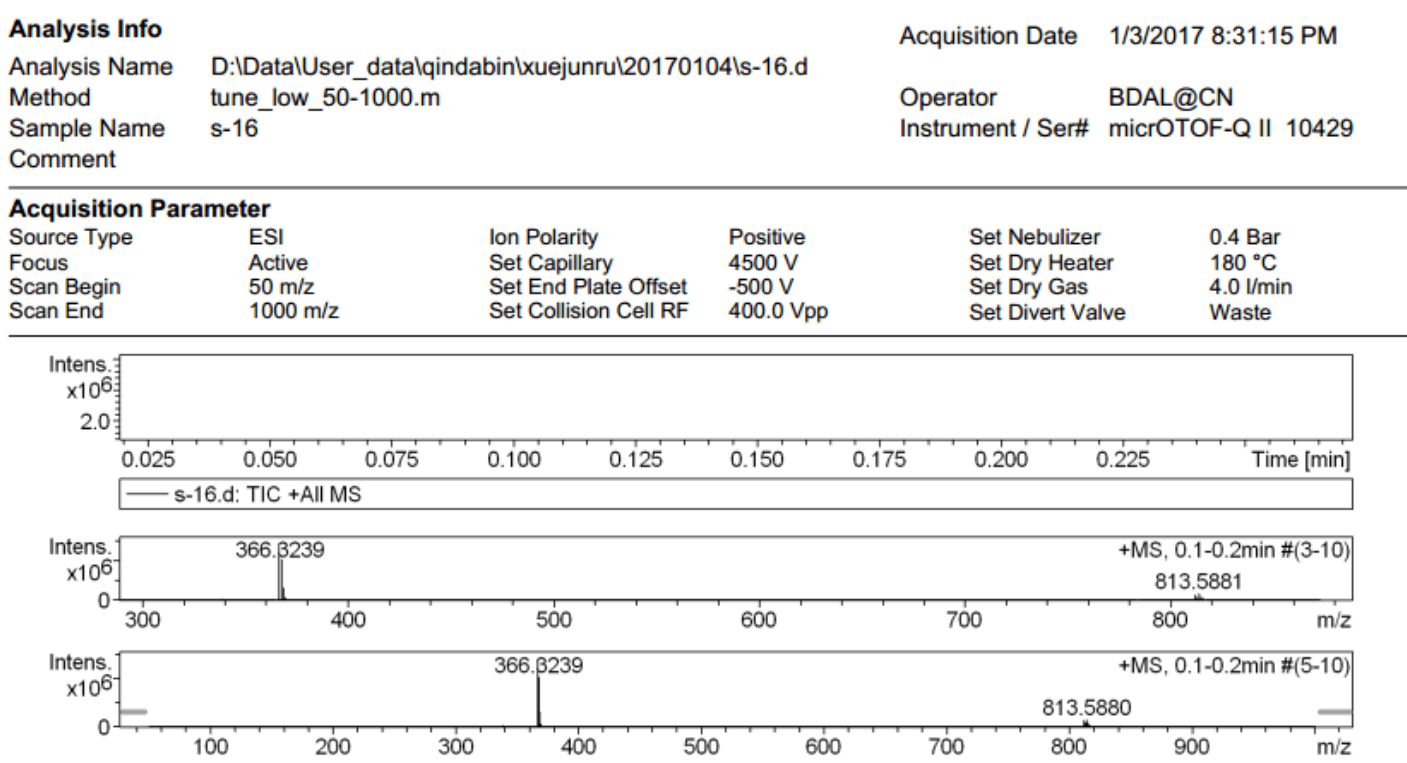

Figure S4 MS report for NTA 
The molecular formula and chemical structures of the organic matter were obtained by analyzing Figs. S1-S4 and Tables S1-S3. The organic matter $\mathrm{M}=366.3239$. $3085.21\left(\mathrm{~cm}^{-1}\right), 1640.62\left(\mathrm{~cm}^{-1}\right), 1473.01\left(\mathrm{~cm}^{-1}\right),{ }^{1} 827.61\left(\mathrm{~cm}^{-1}\right)$ and other FT-IR peaks indicate that the organic compound has a thiophene ring. Results of binding to ${ }^{1} \mathrm{H}$ NMR and ${ }^{13} \mathrm{C}$ NMR spectra: ${ }^{1} \mathrm{H}$ NMR (ppm): $\delta$ 0.91-0.88 (t, 3H), 1.36-1.27 (m, 26H), $1.81(\mathrm{~m}, 2 \mathrm{H}), 3.50-3.46(\mathrm{t}, 2 \mathrm{H}),{ }^{2}{ }^{13} \mathrm{C}$ NMR (ppm): $\delta$ 63.69 31.90, 29.73, 29.67, $29.63,29.58,29.53,29.45,29.40,29.34,29.25,29.17,26.30,22.92,22.67,14.14$ indicate a C-16 alkyl linear group attached to $\mathrm{N} ;{ }^{1} \mathrm{H}$ NMR (ppm): $\delta 5.13(\mathrm{~s}, 2 \mathrm{H}),{ }^{13} \mathrm{C}$ NMR (ppm): $\delta 61.63$ represent a methylene group attached to the $\mathrm{N}$ and thiophene ring; ${ }^{1} \mathrm{H}$ NMR (ppm): $\delta$ 7.96-7.95 $(\mathrm{m}, 1 \mathrm{H}), 7.45-7.43(\mathrm{~m}, 1 \mathrm{H}), 7.33-7.32(\mathrm{~m}, 1 \mathrm{H}),{ }^{13} \mathrm{C}$ NMR (ppm): $\delta 132.19,130.13,127.93,127.40$ represent a meta-substituted thiophene ring; ${ }^{3}{ }^{1} \mathrm{H}$ NMR (ppm): $\delta 3.31(\mathrm{~s}, 6 \mathrm{H}),{ }^{13} \mathrm{C}$ NMR (ppm): $\delta 49.69$ indicate two equivalent methyl groups at a mirror-symmetric position attached to N. According to the above analysis and experimental content, it is proved that the synthesized organic matter is N-alkyl-N, N-dimethyl-N-(3-thienylmethylene) ammonium bromides, and the chemical formula is: $\left[\mathrm{C}_{23} \mathrm{H}_{42} \mathrm{SN}\right] \mathrm{Br}$. 


\section{REFERENCE}

1. Silva, A. J. C.; Ferreira, S. M. F.; Santos, D. d. P.; Navarro, M.; Tonholo, J.; Ribeiro, A. S., A multielectrochromic copolymer based on pyrrole and thiophene derivatives. Solar Energy Materials and Solar Cells 2012, 103, 108-113.

2. Lara-Ceniceros, A. C.; Rivera-Vallejo, C.; Jiménez-Regalado, E. J., Synthesis and characterization of telechelic polymers obtained by micellar polymerization. Polymer Bulletin 2007, 59, 499-508.

3. Czerwinski, W.; Malinowski, R., The soluble copolymers of polyalkylthiophenes with different molar ratios of co-mers. J. Polym. Eng. 2015, 35, 241-246. 